\title{
Detailed analysis of open clusters: A mass function break and evidence of a fundamental plane
}

\author{
C. Bonatto and E. Bica \\ Universidade Federal do Rio Grande do Sul, Instituto de Física, CP 15051, Porto Alegre 91501-970, RS, Brazil \\ e-mail: charles@if.ufrgs.br
}

Received 10 December 2004 / Accepted 22 March 2005

\begin{abstract}
We derive photometric, structural and dynamical evolution-related parameters of 11 nearby open clusters with ages in the range $70 \mathrm{Myr}$ to $7 \mathrm{Gyr}$ and masses in the range $\approx 400 M_{\odot}$ to $\approx 5300 M_{\odot}$. The clusters are homogeneously analysed by means of $J, H$ and $K_{\mathrm{S}}$ 2MASS photometry, which provides spatial coverage wide enough to properly take into account the contamination of the cluster field by Galaxy stars. Structural parameters such as core and limiting radii are derived from the background-subtracted radial density profiles. Luminosity and mass functions (MFs) are built for stars later than the turnoff and brighter than the 2MASS PSC 99.9\% completeness limit. The total mass locked up in stars in the core and the whole cluster, as well as the corresponding mass densities, are calculated by taking into account the observed stars (evolved and main sequence) and extrapolating the MFs down to the H-burning mass limit, $0.08 M_{\odot}$. We illustrate the methods by analysing for the first time in the near-infrared the populous open clusters NGC 2477 and NGC 2516. For NGC 2477 we derive an age of $1.1 \pm 0.1 \mathrm{Gyr}$, distance from the Sun $d_{\odot}=1.2 \pm 0.1 \mathrm{kpc}$, core radius $R_{\text {core }}=1.4 \pm 0.1 \mathrm{pc}$, limiting radius $R_{\text {lim }}=11.6 \pm 0.7 \mathrm{pc}$ and total mass $m_{\mathrm{tot}} \approx(5.3 \pm 1.6) \times 10^{3} M_{\odot}$. Large-scale mass segregation in NGC 2477 is reflected in the significant variation of the MF slopes in different spatial regions of the cluster, and in the large number-density of giant stars in the core with respect to the cluster as a whole. For NGC 2516 we derive an age of $160 \pm 10 \mathrm{Myr}, d_{\odot}=0.44 \pm 0.02 \mathrm{kpc}, R_{\text {core }}=0.6 \pm 0.1 \mathrm{pc}, R_{\lim }=6.2 \pm 0.2 \mathrm{pc}$ and $m_{\mathrm{tot}} \approx(1.3 \pm 0.2) \times 10^{3} M_{\odot}$. Mass-segregation in NGC 2516 shows up in the MFs. Six of the 11 clusters present a slope break in the MF occurring at essentially the same mass as that found for the field stars in Kroupa's universal IMF. The MF break is not associated to cluster mass, at least in the clusters in this paper. In two clusters the low-mass end of the MF occurs above the MF break. However, in three clusters the MF break does not occur, at least for the mass range $m \geq 0.7 M_{\odot}$. One possibility is dynamical evolution affecting the MF slope distribution. We also search for relations of structural and evolutionary parameters with age and Galactocentric distance. The main results for the present sample are: $(i)$ cluster size correlates both with age and Galactocentric distance; (ii) because of size and mass scaling, core and limiting radii, and core and overall mass correlate; (iii) massive $\left(m \geq 1000 M_{\odot}\right)$ and less-massive clusters follow separate correlation paths on the plane core radius and overall mass; (iv) MF slopes of massive clusters are restricted to a narrow range, while those of the less-massive ones distribute over a wider range. Core and overall MF flattening is related to the ratio $(\tau)$ of age to relaxation time. For large values of $\tau$ the effects of large-scale mass segregation and low-mass stars evaporation can be observed in the MFs. In this sense, $\tau$ appears to characterize the evolutionary state of the clusters. We conclude that appreciable slope flattenings in the overall MFs of the lessmassive clusters take $\sim 6$ times longer to occur than in the core, while in the massive clusters they take a time $\sim 13$ times longer. We investigate cluster parameters equivalent to those determining the fundamental plane of ellipticals. These parameters are: overall mass, projected mass density and core radius. We conclude that in the present sample there is evidence of a fundamental plane. Larger samples are necessary to pin down this issue.
\end{abstract}

Key words. open clusters and associations: general

\section{Introduction}

Open clusters are self-gravitating stellar systems which span a broad range of ages and masses, and present significant variations in linear dimensions and morphology (e.g. Lyngå 1982; Binney \& Merrifield 1998; Tadross et al. 2002). The structure of most open clusters can be described by two subsystems, the dense core and the sparse halo (corona). The stellar content of an open cluster presumably formed at the same time from the same parent interstellar cloud, thus sharing similar initial conditions. Stellar and dynamical evolution, as well as external interactions with Galactic structures, change the morphology and the internal mass distribution of clusters (Bergond et al. 2001). As a consequence of large-scale mass segregation high-mass stars (as well as multiple systems) tend to be more concentrated in the core of evolved clusters while low-mass stars are transferred to the halo. In the oldest clusters (or the less-massive ones) the low-mass stars may evaporate from the cluster and 
get dispersed into the surroundings (de la Fuente Marcos 1998). Consequently, changes which occur along time must be reflected on observational parameters such as core and overall radii, the mass locked up in stars in the core and in the whole cluster, and the mass densities in both structures. In addition, core and overall mass-function slopes change as the clusters evolve (e.g. Kroupa 2001). In this sense, open clusters are excellent laboratories in which models of star formation, stellar evolution and dynamical evolution can be tested both on theoretical grounds and numerical simulations.

The relatively small number of stars (as compared to globular clusters) in open clusters poses some technical challenges to $N$-body simulations, particularly associated to the (i) increased granularity of the gravitational potential; (ii) significant fraction of binaries formed along the evolution; (iii) significant mass loss during the course of the normal stellar evolution, which affects cluster life-time; and (iv) nonspherical spatial shape. A discussion on the above points is in e.g. de la Fuente Marcos \& de la Fuente Marcos (2002). Consequently, it is important to search for correlations among observational parameters in order to better constrain theoretical studies and simulation codes. Accordingly, large spatial coverage, photometric uniformity and a homogeneous analysis method are essential to obtain a consistent set of intrinsic parameters. This point is important since most open clusters are projected against the Galactic plane and their fields may be heavily contaminated by Galactic (field) stars.

Previous searches for correlations involving open cluster parameters can be found in, e.g. Lyngå (1982), which is a compilation of literature data, Tadross et al. (2002), which is based on UBV CCD photometry, and Nilakshi et al. (2002), which is based on star counts extracted from Digital Sky Survey plates. Because of the limited spatial coverage and/or limited photometrical depth of the previous observational techniques, most parameters were restricted to the central regions of the clusters and consequently, they suffered from poor background contamination subtraction, as well as cluster field crowding.

Parameters used in the present work are: (i) Galactocentric distance $\left(d_{\mathrm{GC}}\right)$, which is an external parameter; (ii) cluster age, which is associated to the stellar evolution; ( $i i i)$ core $\left(R_{\text {core }}\right)$ and limiting $\left(R_{\text {lim }}\right)$ radii, which are structural parameters associated to the dynamical evolution; (iv) core and overall mass; ( $v$ ) core and overall projected mass density (in $M_{\odot} \mathrm{pc}^{-2}$ ); (vi) core and overall mass density (in $M_{\odot} \mathrm{pc}^{-3}$ ). Parameters listed in (ii) and (iv) to (vi) are intrinsically associated to the stellar and dynamical evolution. We refer to the whole structure $\left(0 \leq r \leq R_{\lim }\right)$ of a cluster as overall.

For the sake of spatial and photometric uniformity, we employ in the present work $J, H$ and $K_{\mathrm{S}} 2 \mathrm{MASS}^{1}$ photometry. The 2MASS Point Source Catalogue (PSC) is uniform reaching relatively faint magnitudes covering nearly all the sky, allowing a proper background definition even for clusters with large angular sizes. The clusters in the present work have already been studied by us in previous papers (in proper motion and

\footnotetext{
1 The Two Micron All Sky Survey, All Sky data release (Skrutskie et al. 1997), available at http://www.ipac.caltech.edu/2mass/ releases/allsky/
}

dynamical state studies), except for the rich open clusters NGC 2477 and NGC 2516, which are studied in the nearinfrared for the first time and illustrate in detail the present analysis method as well.

Age and Galactocentric distance (derived from the distance from the Sun) are obtained by fitting isochrones to the nearinfrared 2MASS colour-magnitude diagrams (CMDs), which gives as well the foreground colour excess in that direction (e.g. Bica et al. 2004a,b). The statistical significance of these parameters depends directly on the quality and depth of the photometry (Bonatto et al. 2004a, 2005). The core radius is derived by fitting a King model to the radial density profile, while the limiting radius corresponds to the distance from the center where the radial density profile merges with the background level. In this case, deep enough photometry is necessary to produce a radial density profile which includes low main-sequence (MS) stars as well, while spatial coverage is fundamental, since a high background may produce an underestimated cluster dimension (Bica et al. 2004b; Bonatto et al. 2004a, 2005). Wide spatial coverage is important for the dynamically evolved clusters, since a significant fraction of the low-mass content may already have been dispersed into the surroundings (Bonatto \& Bica 2003; Bonatto et al. 2004a, 2005). Core and overall masses are obtained by first deriving the intrinsic luminosity (LF) and mass (MF) functions, which depend essentially on properly taking into account the stellar background distribution, and then integrating the MFs down to the H-burning limit stars. Consequently, depth and coverage are fundamental to properly derive mass, particularly for clusters projected against the disk and/or bulge (Bica et al. 2004b; Bonatto et al. 2005).

In the present work we intend to search for fundamental relations in the parameter space described above. Additionally, since star clusters are, to a first approximation, scaled-down versions of elliptical galaxies, we intend to explore the possibility of an analogous fundamental plane (FP, e.g. Dressler et al. 1987; Djorgovski \& Davis 1987) of open cluster parameters. The parameters which could correspond to those of the FP in ellipticals could be cluster overall and/or core mass, core radius (the analogous of the effective radius in the ellipticals), and the projected mass density. However, the conceptual differences between ellipticals and open clusters, e.g. total population and dynamical evolutionary state, may hinder such eventual relations.

This paper is organized as follows. In Sect. 2 we provide general data on the target clusters. In Sect. 3 we illustrate the analysis methods with NGC 2477 and NGC 2516. Cluster parameters are presented and correlations are discussed in Sect. 4. The MF slope break and dynamical effects on MFs are discussed in Sect. 5. In Sect. 6 we discuss a possible FP in open clusters. Finally, concluding remarks are given in Sect. 7.

\section{The target clusters}

We selected for the present work 9 open clusters already studied by us with 2MASS in previous papers for different goals: M 26 (NGC 6694), NGC 2287, M 48 (NGC 2548), M93 (NGC 2447), NGC 5822, NGC 3680, IC 4651, M 67 (NGC 2682) and NGC 188. Proper motions and binarity in 
Table 1. General data on the target clusters.

\begin{tabular}{|c|c|c|c|c|c|c|c|c|c|c|c|}
\hline \multirow[b]{2}{*}{ Cluster } & \multicolumn{5}{|c|}{ WEBDA } & \multicolumn{5}{|c|}{ Present work } & \multirow{3}{*}{$\begin{array}{c}d_{\mathrm{GC}} \\
(\mathrm{kpc}) \\
(12)\end{array}$} \\
\hline & $\begin{array}{c}\alpha(2000) \\
(\mathrm{hms})\end{array}$ & $\begin{array}{c}\delta(2000) \\
\left({ }^{\circ \prime \prime \prime}\right)\end{array}$ & $\begin{array}{c}\text { Age } \\
(\mathrm{Myr})\end{array}$ & $E(B-V)$ & $\begin{array}{c}d_{\odot} \\
(\mathrm{kpc})\end{array}$ & $\begin{array}{c}\alpha(2000) \\
(\mathrm{hms})\end{array}$ & $\begin{array}{c}\delta(2000) \\
\left({ }^{\prime \prime \prime \prime}\right)\end{array}$ & $\begin{array}{c}\text { Age } \\
(\mathrm{Myr})\end{array}$ & $E(B-V)$ & $\begin{array}{c}d_{\odot} \\
(\mathrm{kpc})\end{array}$ & \\
\hline (1) & (2) & (3) & (4) & $(5)$ & $(6)$ & (7) & $(8)$ & (9) & $(10)$ & $(11)$ & \\
\hline M26 & $18: 45: 18$ & $-09: 23: 00$ & 85 & 0.59 & 1.60 & $18: 45: 14^{\dagger}$ & $-09: 22: 55^{\dagger}$ & $70 \pm 10$ & $0.42 \pm 0.03$ & $1.6 \pm 0.1$ & 6.6 \\
\hline NGC 2516 & 07:58:04 & $-60: 45: 12$ & 113 & 0.10 & 0.41 & 07:58:04 & $-60: 45: 12$ & $160 \pm 10$ & 0.00 & $0.44 \pm 0.02$ & 8.0 \\
\hline NGC 2287 & 06:46:01 & $-20: 45: 24$ & 243 & 0.03 & 0.69 & $06: 46: 01$ & $-20: 45: 24$ & $160 \pm 10$ & 0.00 & $0.8 \pm 0.1$ & 8.5 \\
\hline M 48 & $08: 13: 43$ & $-05: 45: 00$ & 360 & 0.03 & 0.77 & $08: 13: 45^{\dagger}$ & $-05: 46: 42^{\dagger}$ & $360 \pm 40$ & 0.00 & $0.8 \pm 0.1$ & 8.5 \\
\hline M93 & $07: 44: 30$ & $-23: 51: 24$ & 387 & 0.05 & 1.04 & $07: 44: 31^{\dagger}$ & $-23: 50: 42^{\dagger}$ & $400 \pm 50$ & $0.03 \pm 0.01$ & $1.1 \pm 0.1$ & 8.6 \\
\hline NGC 5822 & $15: 04: 21$ & $-54: 23: 48$ & 662 & 0.15 & 0.92 & $15: 04: 21$ & $-54: 23: 48$ & $1000 \pm 100$ & 0.00 & $0.7 \pm 0.1$ & 7.4 \\
\hline NGC 2477 & $07: 52: 10$ & $-38: 31: 48$ & 705 & 0.28 & 1.22 & $07: 52: 18^{\dagger}$ & $-38: 31: 57^{\dagger}$ & $1100 \pm 100$ & $0.06 \pm 0.03$ & $1.2 \pm 0.1$ & 8.4 \\
\hline NGC 3680 & $11: 25: 38$ & $-43: 14: 36$ & 1200 & 0.07 & 0.94 & $11: 25: 38$ & $-43: 14: 36$ & $1600 \pm 100$ & 0.00 & $1.0 \pm 0.1$ & 7.8 \\
\hline IC 4651 & $17: 24: 49$ & $-49: 56: 00$ & 1140 & 0.12 & 0.89 & $17: 24: 52^{\dagger}$ & $-49: 56: 52^{\dagger}$ & $1800 \pm 200$ & 0.00 & $0.9 \pm 0.1$ & 7.2 \\
\hline M 67 & 08:51:18 & $+11: 48: 00$ & 2560 & 0.06 & 0.91 & $08: 51: 16^{\dagger}$ & $+11: 48: 54^{\dagger}$ & $3200 \pm 100$ & 0.00 & $0.9 \pm 0.1$ & 8.7 \\
\hline NGC 188 & $00: 47: 28^{\dagger}$ & $+85: 15: 18^{\dagger}$ & 4300 & 0.08 & 2.05 & $00: 47: 53$ & $+85: 15: 30$ & $7000 \pm 1000$ & 0.00 & $1.7 \pm 0.1$ & 8.9 \\
\hline
\end{tabular}

Table Notes. Colour excesses in Col. 10 are derived from the isochrone fit to the 2MASS CMDs. Uncertainty in Galactocentric distance in Col. 12 is $\approx 0.1 \mathrm{kpc}$ for all clusters. Uncertainties in Cols. 9 to 12 are derived from the isochrone fit. $\left({ }^{\dagger}\right)$ Coordinates optimized to maximize the number-density of stars in the cluster center (Sect. 3).

M 26, NGC 2287, M 48, M 93, NGC 5822, NGC 3680, IC 4651 and M 67 were investigated in Bica \& Bonatto (2005). The advanced dynamical state of NGC 3680 was analysed in Bonatto et al. (2004a). Mass segregation in M 67 was studied in Bonatto $\&$ Bica (2003). The dynamical state of the old open cluster NGC 188 was investigated in Bonatto et al. (2005).

The populous open clusters NGC 2477 and NGC 2516 are discussed in Sect. 3. The clusters above span a wide age range, which is important since age is a fundamental factor associated to dynamical evolution. General data for the above open clusters are given in Table 1. The data in Cols. 2-6 are from the WEBDA ${ }^{2}$ open cluster database (Mermilliod 1996). In Cols. 7-12 we list parameters derived from the 2MASS photometry.

We indicate below papers and results relevant to the present work, discriminated by open cluster.

M 26: Battinelli et al. (1994) give a limiting radius of $R_{\lim } \approx$ $3.3 \mathrm{pc}$ and a total stellar mass of $m_{\mathrm{tot}} \approx 240 M_{\odot}$.

NGC 2516: Battinelli et al. (1994) give $R_{\lim } \approx 1.9 \mathrm{pc}$ and $m_{\mathrm{tot}} \approx 282 M_{\odot}$. Bergond et al. (2001) give $d_{\odot} \approx 0.4 \mathrm{kpc}$, an age of $\approx 113 \mathrm{Myr}, R_{\text {tidal }}=1.9 \mathrm{pc}$ and $m_{\mathrm{tot}} \approx 170 M_{\odot}$. Tadross et al. (2002) give $d_{\odot} \approx 0.4 \mathrm{kpc}$, age $\approx 63 \mathrm{Myr}, R_{\mathrm{lim}} \approx 1.8 \mathrm{pc}$, $d_{\mathrm{GC}} \approx 8.5 \mathrm{kpc}$ and $m_{\mathrm{tot}} \approx 36 M_{\odot}$. Jeffries et al. (2001) estimate an age of $\approx 150 \mathrm{Myr}$ and total mass of $m_{\mathrm{tot}} \approx 1240-1560 M_{\odot}$. They also detected a sharp change in the MF slope for stars with $0.3 \leq m\left(M_{\odot}\right) \leq 0.7$.

NGC 2287: Battinelli et al. (1994) give $R_{\text {lim }} \approx 4.4 \mathrm{pc}$ and $m_{\mathrm{tot}} \approx 195 M_{\odot}$. Bergond et al. (2001) included this object in a study of the gravitational tidal effects on open clusters.

\footnotetext{
${ }^{2}$ http://obswww.unige.ch/webda
}

They give a distance from the Sun of $d_{\odot} \approx 0.7 \mathrm{kpc}$, an age of $\approx 240 \mathrm{Myr}$, a tidal radius of $R_{\mathrm{tidal}}=4.1 \mathrm{pc}$ and a total stellar mass of $m_{\mathrm{tot}} \approx 300 M_{\odot}$.

M 48: Bergond et al. (2001) give $d_{\odot} \approx 0.8 \mathrm{kpc}$, an age of $\approx 360 \mathrm{Myr}$ and $R_{\mathrm{tidal}}=4.8 \mathrm{pc}$.

M 93: Nilakshi et al. (2002) derived $R_{\text {core }} \approx 0.5$ pc and $R_{\lim } \approx$ $3.3 \mathrm{pc}$.

NGC 5822: Battinelli et al. (1994) give $m_{\mathrm{tot}} \approx 251 M_{\odot}$.

NGC 2477: Based on $U B V$ photometry of 83 red-giant candidates in the field of NGC 2477, Eigenbrod et al. (2004) derived a core radius $R_{\text {core }}=1.8 \mathrm{pc}$ and a tidal radius $R_{\text {tidal }}=8.1 \mathrm{pc}$. They also estimate that the total mass locked up in stars more massive than $\approx 1 M_{\odot}$ must be larger than $5400 M_{\odot}$. A break in the mass function in the lower main sequence at $m \approx 0.27 M_{\odot}$ was suggested by von Hippel et al. (1996). Nilakshi et al. (2002) derived $R_{\text {core }} \approx 2.2$ pc and $R_{\text {lim }} \approx 9.1$ pc. Tadross et al. (2002) give $d_{\odot} \approx 1.4 \mathrm{kpc}$, age $\approx 1 \mathrm{Gyr}, R_{\mathrm{lim}} \approx 5.5 \mathrm{pc}$, $d_{\mathrm{GC}} \approx 9.0 \mathrm{kpc}$ and $m_{\mathrm{tot}} \approx 2100 M_{\odot}$.

NGC 3680: Bonatto et al. (2004a) studied this dynamically advanced open cluster and derived $d_{\odot} \approx 1 \mathrm{kpc}$, age $\approx 1.6 \mathrm{Gyr}$, $R_{\text {core }} \approx 0.7 \mathrm{pc}, R_{\mathrm{lim}} \approx 6.4 \mathrm{pc}$ and $m_{\mathrm{tot}} \approx 550 M_{\odot}$.

IC 4651: Meibom et al. (2002) derived an age of $\approx 1.7 \mathrm{Gyr}$, $R_{\text {tidal }} \approx 5.8 \mathrm{pc}$ and a present total mass of $\sim 630 M_{\odot}$; they also present evidence of moderate mass segregation. Tadross et al. (2002) give $d_{\odot} \approx 0.8 \mathrm{kpc}$, age $\approx 2.2 \mathrm{Gyr}, R_{\mathrm{lim}} \approx 1.4 \mathrm{pc}, d_{\mathrm{GC}} \approx$ $7.8 \mathrm{kpc}$ and $m_{\mathrm{tot}} \approx 100 M_{\odot}$. 
M 67: Battinelli et al. (1994) give $m_{\mathrm{tot}} \approx 407 M_{\odot}$. Bonatto \& Bica (2003) derived an age of $\approx 3.2 \mathrm{Gyr}, d_{\odot} \approx 0.9 \mathrm{kpc}, R_{\text {core }}=$ $1.2 \mathrm{pc}$ and $R_{\mathrm{lim}} \approx 1.8 \mathrm{pc}$. They also detected mass segregation. Mass segregation in M 67 was also detected by Sarajedini et al. (1999). Montgomery et al. (1993) estimate a mass of $\approx 800 M_{\odot}$. Detailed $N$-body modelling of the dynamical and stellar evolution of M 67 was carried out by Hurley et al. (2001); they estimate a much larger initial mass for M 67. Tadross et al. (2002) give $d_{\odot} \approx 0.8 \mathrm{kpc}$, age $\approx 4 \mathrm{Gyr}, R_{\mathrm{lim}} \approx 3.2 \mathrm{pc}, d_{\mathrm{GC}} \approx 9.1 \mathrm{kpc}$ and $m_{\text {tot }} \approx 820 M_{\odot}$.

NGC 188: Bonatto et al. (2005) derived an age of $\approx 7 \mathrm{Gyr}$, $d_{\odot} \approx 1.7 \mathrm{kpc}, R_{\text {core }} \approx 1.3 \mathrm{pc}, R_{\text {tidal }} \approx 21 \mathrm{pc}$ and a total mass of $\approx 4000 M_{\odot}$; they detected strong mass segregation. Mass segregation was also detected by Sarajedini et al. (1999). Nilakshi et al. (2002) derived $R_{\text {core }} \approx 2.1$ pc and $R_{\text {lim }} \approx 6.5$ pc. Tadross et al. (2002) give $d_{\odot}=1.5 \mathrm{kpc}$, age $\approx 5 \mathrm{Gyr}, R_{\mathrm{lim}} \approx 3.1 \mathrm{pc}$, $d_{\mathrm{GC}} \approx 9.4 \mathrm{kpc}$ and an exceedingly low mass of $\approx 208 M_{\odot}$.

The near-infrared CMDs and radial density profiles of M 26, NGC 2287, M 48, M 93, NGC 5822, NGC 3680, IC 4651 and M 67 are shown in Bica \& Bonatto (2005). For NGC 188 they are in Bonatto et al. (2005).

Comparing WEBDA compilation with the present results (Table 1), ages and distances from the Sun agree in general. However, the colour excesses $E(B-V)$ tend to be smaller when derived from the near-infrared, as discussed in Bica \& Bonatto (2005).

\section{The populous open clusters NGC 2477 and NGC 2516}

\subsection{The intermediate-age open cluster NGC 2477}

According to WEBDA, the central coordinates of NGC 2477 are (J2000) $\alpha=07^{\mathrm{h}} 52^{\mathrm{m}} 10^{\mathrm{s}}$, and $\delta=-38^{\circ} 31^{\prime} 48^{\prime \prime}$. However, the corresponding radial density profile (built with a step in radius of $\Delta r=1^{\prime}$ ) presented a dip for $r=0^{\prime}$. We searched for a new center by examining histograms for the number of stars in bins of right ascension and declination. The resulting coordinates which maximize the density of stars at the center are (J2000) $\alpha=07^{\mathrm{h}} 52^{\mathrm{m}} 18^{\mathrm{s}}$, and $\delta=-38^{\circ} 31^{\prime} 57^{\prime \prime}$, corresponding to $\ell=$ $253.58^{\circ}$ and $b=-5.82^{\circ}$. In what follows we refer to these coordinates as the center of NGC 2477.

\subsubsection{The 2MASS photometry and near-infrared CMD}

The VizieR ${ }^{3}$ tool has been used to extract 2MASS photometry of the stars in a circular area with radius $r=60^{\prime}$ centered on the optimized coordinates (Table 1). 2MASS photometric uncertainties as a function of magnitude are discussed in Soares \& Bica (2002) and Bonatto et al. (2005).

Because of the moderately high Galactic latitude $\left(b \approx-6^{\circ}\right)$, the field of NGC 2477 presents limited background contamination. This can be seen in Fig. 1 in which we show the $J \times(J-H)$ CMD of the central $\left(r=10^{\prime} \sim 3.6 \mathrm{pc}\right)$ region of NGC 2477

\footnotetext{
${ }^{3}$ http://vizier.u-strasbg.fr/viz-bin/ VizieR?-source=II/246
}

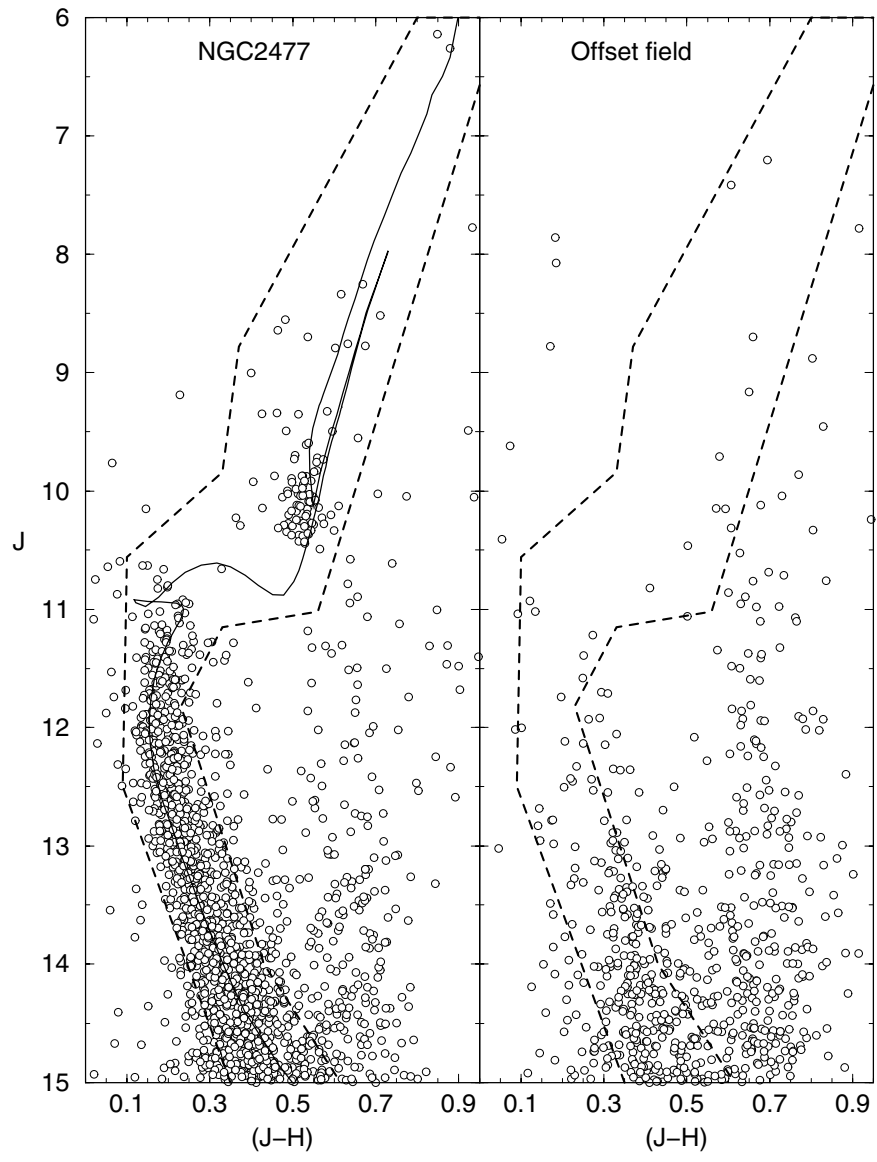

Fig. 1. Left panel: $J \times(J-H) \mathrm{CMD}$ of the central $10^{\prime}(\sim 3.6 \mathrm{pc})$ of NGC 2477 with the best-fit Padova isochrone (age $~ 1.1 \mathrm{Gyr}$ ) superimposed. Note the densely populated main sequence and rich giant clump. Right panel: CMD of the corresponding (same area) offset field. The colour-magnitude filter is shown in both panels.

(left panel) compared with the corresponding, same area, offset field (right panel). To match the areas of both CMDs in Fig. 1 the offset field has been obtained by extracting stars in the outermost ring, located at 59.16 $\leq r \leq 60^{\prime}$, which represents essentially the Galactic background/foreground stellar contribution. The CMD of the central region of NGC 2477 presents a well-defined morphology including a densely populated MS up to the turnoff and a rich giant clump that strongly contrasts with the CMD of the offset field. We show in both panels the colour-magnitude filter which is used to increase membership probability by discarding stars with far-off colours with respect to the isochrone fit (see below).

Cluster parameters have been derived by fitting solar metallicity Padova isochrones (Girardi et al. 2002) computed with the 2MASS $J, H$ and $K_{\mathrm{S}}$ filters ${ }^{4}$, to the observed CMD in Fig. 1. The 2MASS transmission filters produced isochrones very similar to the Johnson ones, with differences of at most 0.01 in $(J-H)$ (Bonatto et al. 2004b). For reddening and absorption transformations we use $R_{V}=3.2$, and the relations $A_{J}=0.276 \times A_{V}$ and $E(J-H)=0.33 \times E(B-V)$, according to Dutra et al. (2002) and references therein. The best-fit

\footnotetext{
${ }^{4}$ http://pleiadi.pd.astro.it/isoc_photsys.01/ isoc_photsys.01.html
} 
for NGC 2477 uses the 1.12 Gyr isochrone. Taking into account the uncertainties associated to the isochrone fit we derive for NGC 2477 an age of $1.1 \pm 0.1 \mathrm{Gyr}$, a colour excess $E(J-H)=0.02 \pm 0.01$ which corresponds to $E(B-V)=$ $0.06 \pm 0.03$, a distance modulus $(m-M)_{J}=10.5 \pm 0.1$, and a distance from the Sun $d_{\odot}=1.23 \pm 0.06 \mathrm{kpc}$. With this value the Galactocentric distance of NGC 2477 turns out to be $d_{\mathrm{GC}}=8.4 \pm 0.1 \mathrm{kpc}$, using $8.0 \mathrm{kpc}$ as the distance of the Sun to the center of the Galaxy (Reid 1993).

\subsubsection{Structural parameters}

Structural parameters of NGC 2477 have been derived by means of the radial density profile, defined as the projected number of stars per area around the cluster center. Before counting stars we applied the colour filter shown in Fig. 1 to the CMD of the cluster (for stars with distance to the center from $0^{\prime}$ to $60^{\prime}$ ) in order to discard most of the contamination by Milky Way stars. This procedure has been previously applied in the analysis of the open clusters M 67 (Bonatto \& Bica 2003), NGC 188 (Bonatto et al. 2005) and NGC 3680 (Bonatto et al. 2004a). To further minimize the probability of background contamination, spurious detections and the increase of photometric uncertainties at faint magnitudes we restricted the radial density analysis to filtered stars with $J \leq 14.5$, corresponding to $M_{J} \leq 4.0$ and mass $m \geq 0.95 M_{\odot}$ (Sect. 3.1.3). At $J=14.5$ the photometric uncertainties amount to $\epsilon_{J} \approx 0.04$ (Bonatto et al. 2005). The radial density profile was obtained by counting stars inside concentric annuli with a step of $1.0^{\prime}$ in radius. The background contribution level corresponds to the average number of stars included in the ring located at $40^{\prime} \leq r \leq 60^{\prime}$ $(14.3 \leq r(\mathrm{pc}) \leq 21.5)$. The above procedures were applied to the filtered CMD.

The resulting radial density profile of NGC 2477 is shown in Fig. 2. For absolute comparison between clusters we scale the radius in the abscissa in parsecs, and the number density of stars in the ordinate in stars $\mathrm{pc}^{-2}$ using the distance derived in Sect. 3.1.1. The statistical significance of the profile is reflected in the relatively small $1 \sigma$ Poisson error bars.

Structural parameters of NGC 2477 were derived by fitting the two-parameter King (1966a) surface density profile to the background-subtracted radial distribution of stars. The two-parameter King model essentially describes the intermediate and central regions of normal clusters (King 1966b; Trager et al. 1995). The fit was performed using a nonlinear leastsquares fit routine which uses the errors as weights. The best-fit solution is shown in Fig. 2 superimposed on the radial density profile. From the fit we derived a core radius $R_{\text {core }}=1.4 \pm$ $0.1 \mathrm{pc}$ and a projected central density of stars of $\sigma_{0} \approx 70 \pm$ 7 stars $\mathrm{pc}^{-2}$. Our value of $R_{\text {core }}$ is somewhat smaller than $R_{\text {core }}=1.8 \mathrm{pc}$ of Eigenbrod et al. (2004) and $R_{\text {core }}=2.2 \mathrm{pc}$ of Nilakshi et al. (2002). The present central density of stars is $\sim 3 \times$ that of Nilakshi et al. (2002).

Within uncertainties the two-parameter King model reproduces well the radial density profile in NGC 2477. Since it follows from an isothermal (virialized) sphere, the close similarity of the radial distribution of stars in NGC 2477 with a King

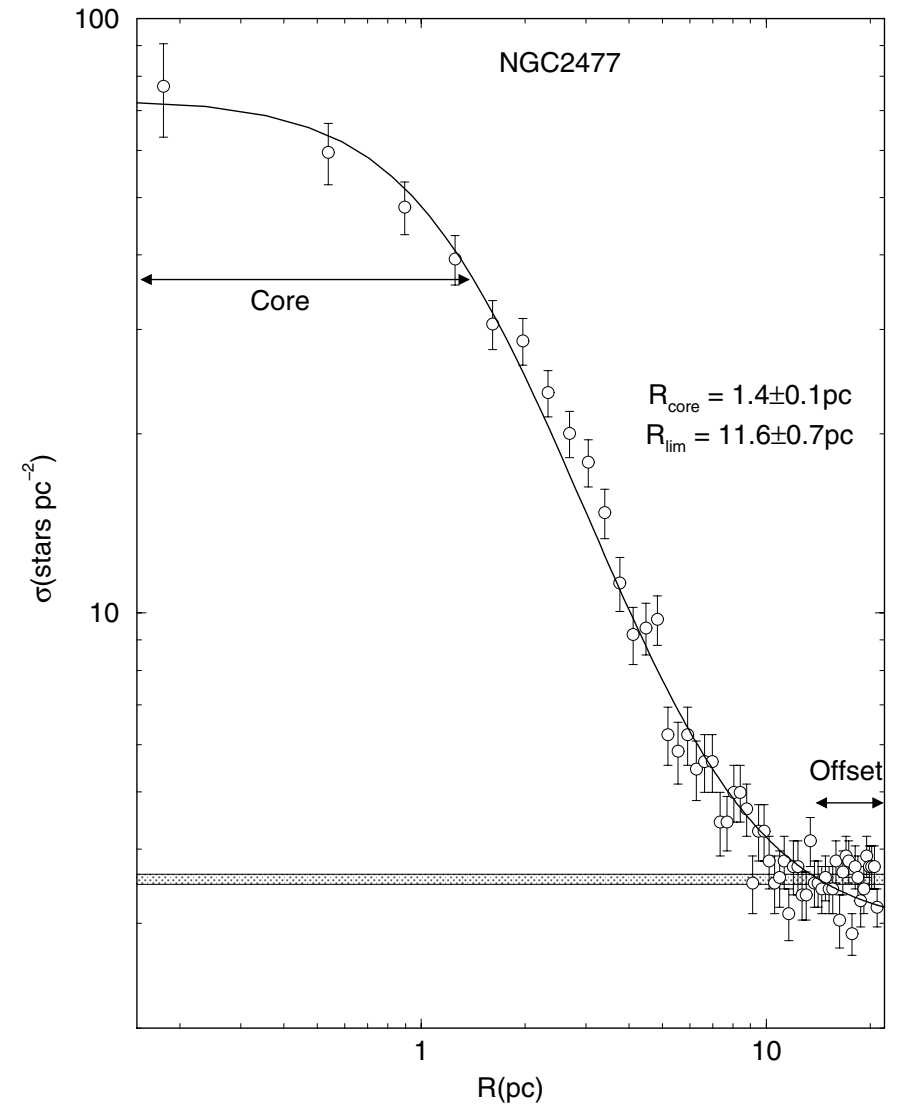

Fig. 2. Projected radial density profile of stars in NGC 2477. The average background level is shown as the narrow shaded rectangle; $1 \sigma$ Poisson errors are also shown. The solid line shows the two-parameter King model fit to the radial profile. The extent of the core region and that of the offset field are indicated.

profile suggests that despite its intermediate age, this cluster has evolved into an advanced dynamical state. We will return to this point in Sect. 3.1.3.

Considering the radial density profile fluctuations with respect to the background level, we can define a limiting radius $\left(R_{\lim }\right)$ for the cluster, in the sense that for regions beyond $R_{\text {lim }}$, the null-contrast between cluster and background star density would produce large Poisson errors and consequently, meaningless results. Thus, for practical purposes, the bulk of the cluster's stars are contained within $R_{\text {lim. }}$. In the case of NGC 2477 the limiting radius turns out to be $R_{\text {lim }}=11.6 \pm$ 0.7 pc. Finally, we derived the tidal radius $\left(R_{\text {tidal }}\right)$ by fitting the three-parameter King (1962) model to the backgroundsubtracted radial distribution of stars in Fig. 2. The resulting $R_{\text {tidal }}=16 \pm 2 \mathrm{pc}$ is $\sim 33 \%$ larger than $R_{\text {lim }}$. Our value of $R_{\text {tidal }}$ is $\sim 2$ times larger than the values given by Eigenbrod et al. (2004) and Nilakshi et al. (2002), and a factor of $\sim 3$ larger than that of Tadross et al. (2002). The significant differences between our values of $R_{\text {core }}$ and $R_{\text {tidal }}$ and those of previous works may be accounted for by the depth of the 2MASS photometry, the proper background definition and the use of a colour-magnitude filter. 


\subsubsection{Mass functions}

The populous nature of NGC 2477 provides an opportunity to study in detail with a high statistical significance the spatial distribution of mass functions $\left(\phi(m)=\frac{\mathrm{d} N}{\mathrm{~d} m}\right)$. Based on the King profile fit and taking into account the features present in the radial density profile of NGC 2477 (Fig. 2) we selected the following regions: (i) $0.0 \leq r(\mathrm{pc}) \leq 1.4$ (core), (ii) $1.4 \leq r(\mathrm{pc}) \leq$ 5.2 , (iii) $5.2 \leq r(\mathrm{pc}) \leq 11.6$ and (iv) $0.0 \leq r$ (pc) $\leq 11.6$ (overall). Regions ( $i i)$ and (iii) are the halo of NGC 2477. In order to maximize the significance of background counts, we consider as offset field the outermost ring at $14.3 \mathrm{pc} \leq r \leq 21.5 \mathrm{pc}$, which lies $\sim 2.7 \mathrm{pc}$ beyond the cluster's limiting radius.

Although relatively small, the Galactic stars contamination of the CMD of NGC 2477 must be taken into account in order to derive the intrinsic luminosity and mass distributions of the member stars. To do this we first apply the colour filter (Fig. 1) to both cluster and offset field CMDs. The filtering process takes into account most of the background, leaving a residual contamination. We deal with this residual contamination statistically by building the LFs for each cluster region and offset field. We build LFs for the three 2MASS bands independently, taking into account the $99.9 \%$ Point Source Catalogue Completeness Limit ${ }^{5}$. Consequently, the faint magnitude limit of each LF is $J=15.8, H=15.1$ and $K_{\mathrm{S}}=14.3$, respectively. We take the turnoff as the bright limit to avoid inconsistencies in the mass-luminosity relation. For each 2MASS band we build a LF by counting stars in magnitude bins from the respective faint magnitude limit to the turnoff, both for each cluster region and offset field. Considering that the solid angle of the offset field is different from that of a given cluster region, we multiply the offset field LF by a numerical factor so that the solid angles match. The intrinsic LF of each cluster region is obtained by subtracting the respective (i.e. solid angle-corrected) offset field LF from that of the cluster region. Finally, the intrinsic LFs are transformed into MFs using the mass-luminosity relation obtained from the $1.12 \mathrm{Gyr}$ Padova isochrone and the distance modulus $(m-M)_{J}=10.5$. Remark that these procedures are repeated independently for the three 2MASS bands. The final MF of a given cluster region is produced by combining the $J, H$ and $K_{\mathrm{S}}$ MFs into a single MF. The resulting spatial MFs of NGC 2477, covering the mass range $0.76 \leq m\left(M_{\odot}\right) \leq 1.95$, are shown in Fig. 3 .

The MFs present a break followed by slope flattening for masses in the range $0.76 \leq m\left(M_{\odot}\right) \leq 1.08$, which is noticeable particularly in the core (panel d) and in the region $1.4 \leq r(\mathrm{pc}) \leq$ 5.2 (panel c). We note that von Hippel et al. (1996), based on deep V and I HST and WFPC2 photometry of the central parts of NGC 2477, described a break in the MF, but occurring at a lower mass value, $m \approx 0.27 M_{\odot}$ (Sect. 5).

Bearing in mind the MF break at $m_{\text {break }}=1.08 M_{\odot}$ we fit the function $\phi(m) \propto m^{-(1+\chi)}$ in the mass ranges $0.76 \leq$ $m\left(M_{\odot}\right) \leq 1.08$ and $1.08 \leq m\left(M_{\odot}\right) \leq 1.95$. The resulting fits are shown in Fig. 3, and the MF slopes of each mass range are given in Cols. 4 and 5 of Table 2, respectively.

\footnotetext{
5 Corresponding to the Level 1 Requirement, according to http://www .ipac.caltech.edu/2mass/releases/allsky/doc/ sec6_5a1.html
}

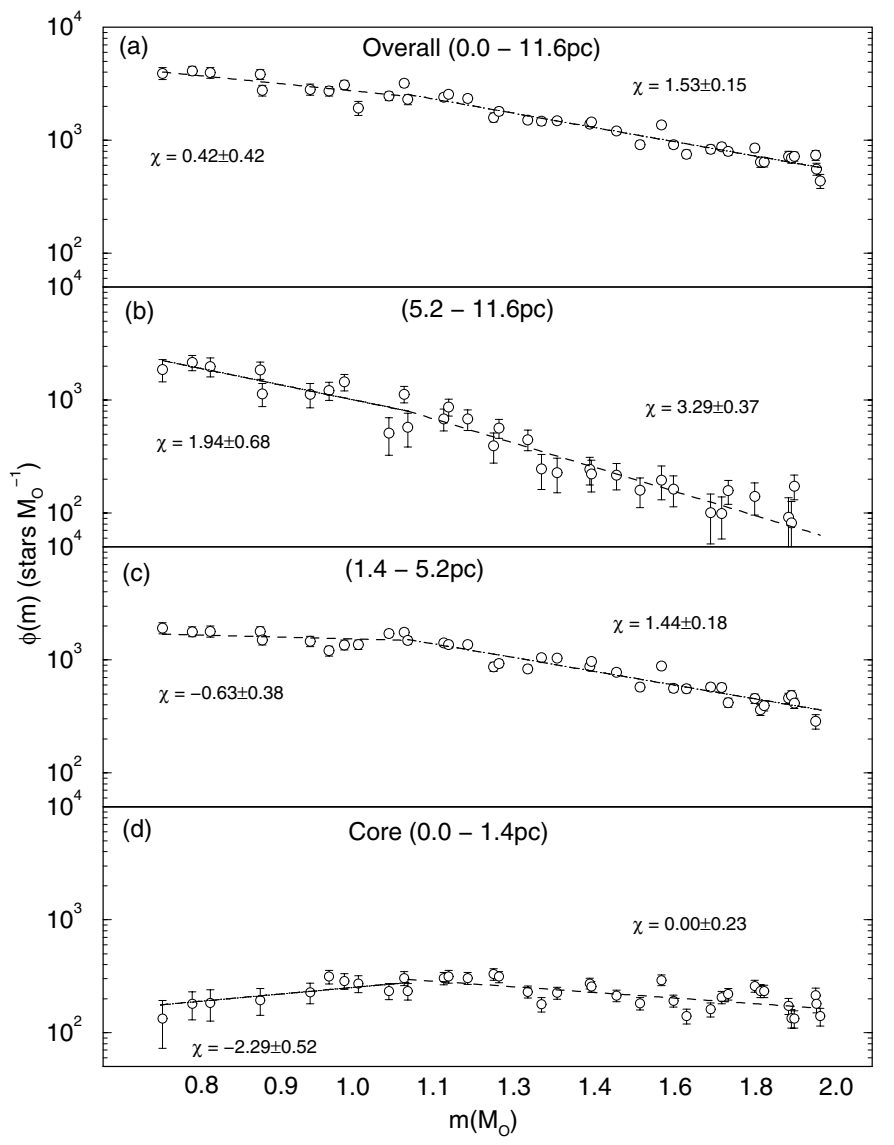

Fig. 3. Mass functions in different spatial regions of NGC 2477. Each panel contains MFs derived from the $J, H$ and $K_{\mathrm{S}}$ 2MASS photometry. A break in the MFs at $m \approx 1.08 M_{\odot}$ can be seen particularly in panels c) and d). MF fits $\left(\phi(m) \propto m^{-(1+\chi)}\right)$ for each mass range are shown as dashed lines. The respective MF slopes are given. Note that the flattest (panel d) and steepest (panel b) MFs coincide spatially in both mass ranges.

We provide in Table 2 parameters derived from the LFs and MFs. The number of evolved stars (Col. 2) is calculated by integrating the intrinsic LFs for magnitudes brighter than the turnoff. Multiplying this by the mass at the turnoff $\left(1.98 M_{\odot}\right)$ gives an estimate of the evolved-star mass (Col. 3). This procedure produces a realistic value of the number of member evolved stars because the background contamination has already been statistically subtracted from the LF. The MF slopes in the two mass ranges are in Cols. 4 and 5. The number of MS stars and corresponding stellar mass are derived by integrating the MF from the faint magnitude limit to the turnoff. We add to these the corresponding values of the number and mass of evolved stars to derive the total number of observed stars (Col. 6), observed mass (Col. 7), projected mass density (Col. 8) and mass density (Col. 9).

An estimate of the total mass locked up in stars in NGC 2477 was made by taking into account all stars from the turnoff down to the H-burning mass limit, $0.08 M_{\odot}$. We do this by directly extrapolating the low-mass MFs $(0.76 \leq$ $\left.m\left(M_{\odot}\right) \leq 1.08\right)$ down to $0.08 M_{\odot}$, except for the region $5.2 \leq r(\mathrm{pc}) \leq 11.6$ for which the slope is $\chi=1.9 \pm 0.7$. In this case we assume the universal Initial Mass Function 
Table 2. Data derived from the mass functions in NGC 2477 and NGC 2516.

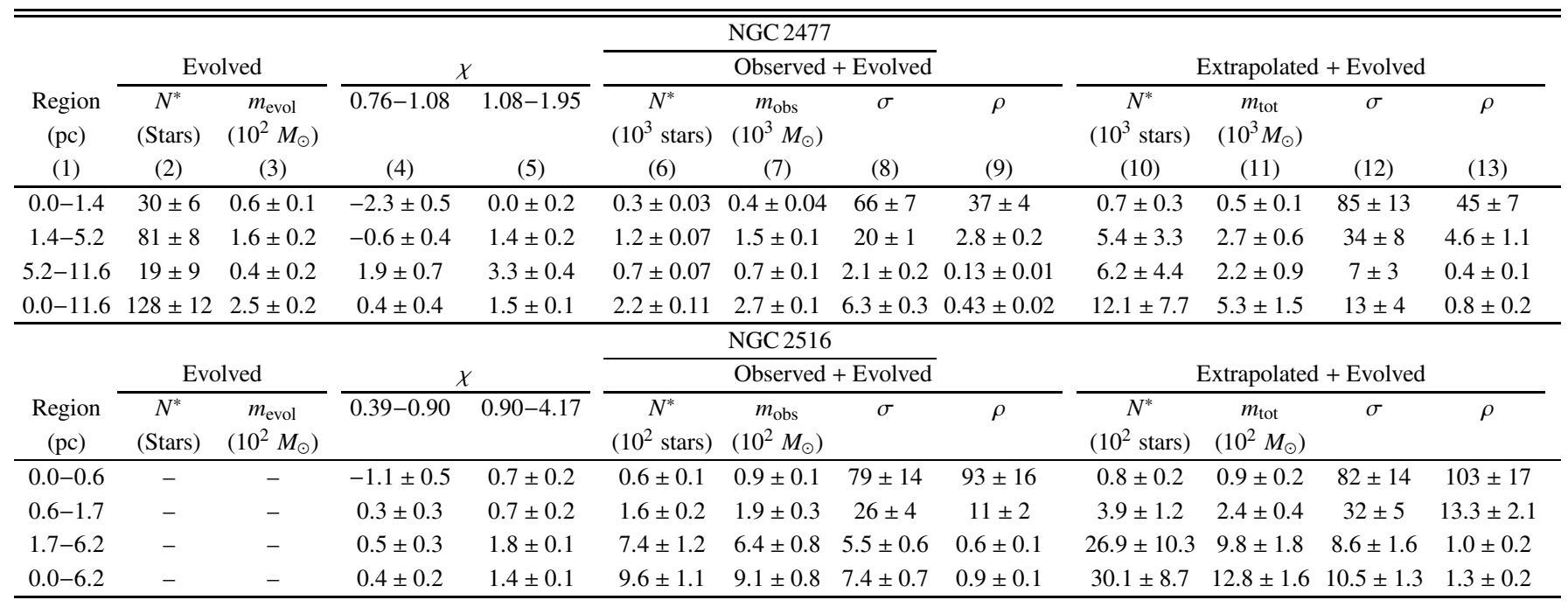

Notes. Columns 4 and 5 give the MF slopes derived for the low-mass and high-mass MS ranges. Notice that the low-mass MS ranges are $0.76 \leq m\left(M_{\odot}\right) \leq 1.08$ and $0.39 \leq m\left(M_{\odot}\right) \leq 0.90$, respectively for NGC 2477 and NGC 2516. The high-mass MS ranges are $1.08 \leq m\left(M_{\odot}\right) \leq 1.50$ and $0.90 \leq m\left(M_{\odot}\right) \leq 4.17$. The mass of the evolved stars is included in $m_{\mathrm{obs}}(\mathrm{Col} .7)$ and $m_{\mathrm{tot}}(\mathrm{Col} .11)$. Units of $\sigma$ (Cols. 8 and 12) and $\rho$ (Cols. 9 and 13) are $M_{\odot} \mathrm{pc}^{-2}$ and $M_{\odot} \mathrm{pc}^{-3}$, respectively.

(IMF) of Kroupa (2001), in which $\chi=0.3 \pm 0.5$ for the range $0.08 \leq m\left(M_{\odot}\right) \leq 0.5$. In the range $0.5 \leq m\left(M_{\odot}\right) \leq 0.76$ we use our value of $\chi$ which, within the uncertainty is similar to the value presented in Kroupa (2001) for the equivalent mass range. The resulting extrapolated values of the number of stars and extrapolated mass (added to the corresponding values of the number and mass of evolved stars) are given respectively in Cols. 10 and 11 of Table 2. The extrapolated projected and mass densities are in Cols. 12 and 13.

The total mass locked up in stars in the core of NGC 2477 results in $m_{\text {tot }}($ core $) \approx(5.2 \pm 0.1) \times 10^{2} M_{\odot}$, nearly $25 \%$ larger than the mass corresponding to the observed stars. The total overall mass is $m_{\mathrm{tot}}($ overall $) \approx(5.3 \pm 1.5) \times 10^{3} M_{\odot}$, which is in close agreement with the mass estimate (for stars with $m \geq$ $1 M_{\odot}$ ) of Eigenbrod et al. (2004) and 2.5 times larger than the estimate of Tadross et al. (2002). The extrapolated overall mass is nearly twice the mass stored in the observed stars.

\subsubsection{Mass segregation}

Within the uncertainty, the overall MF slope $(\chi=1.5 \pm 0.1)$ in the mass range $1.08 \leq m\left(M_{\odot}\right) \leq 1.95$ is similar to a standard Salpeter $(\chi=1.35)$ IMF. However, the MF slope presents large variations in the inner regions, being flat $(\chi=0.0 \pm$ $0.2)$ in the core and steep $(\chi=3.3 \pm 0.24)$ in the region $5.2 \leq r(\mathrm{pc}) \leq 11.6$. MF slope flattening from the outskirts to the core is detected in the mass range $0.76 \leq m\left(M_{\odot}\right) \leq 1.08$ as well. Similarly to the mass range $1.08 \leq m\left(M_{\odot}\right) \leq 1.95$ the flattest MF $(\chi=-2.3 \pm 0.5)$ occurs in the core, while the steepest MF $(\chi=1.9 \pm 0.7)$ occurs in the region $5.2 \leq r(\mathrm{pc}) \leq 11.6$. This fact reflects the advanced dynamical state of NGC 2477 , particularly the effects of large-scale mass segregation, in the sense that low-mass stars originally in the core are being transferred to the cluster's outskirts while massive stars accumulate in the core. This produces a flat MF in the core and a steep one in the halo.

Another evidence pointing to large-scale mass segregation is the large difference in the number-density of giants in each cluster region with respect to that of MS stars. We assume here as giants the stars brighter than the turnoff (with mass $\left.m \geq 1.98 M_{\odot}\right)$ and as MS stars those with mass in the range $0.08 \leq m\left(M_{\odot}\right) \leq 1.98$. For the giants the number-density ratio is $\frac{\rho^{*} \text { (core) }}{\rho^{*} \text { (overall) }} \sim 130$, while for the MS stars the ratio drops to $\frac{\rho^{*} \text { (core) }}{\rho^{*} \text { (overall) }} \sim 30$. This means that in the core of NGC 2477 the number-density of giants with respect to MS stars is $\sim 4$ larger than in the cluster as a whole. The situation gets reversed in the region $5.2 \leq r(\mathrm{pc}) \leq 11.6$, where the giants and MS numberdensity ratios are, respectively $\frac{\rho^{*}(\text { core })}{\rho^{*}(\text { overall })} \sim 0.15$ and $\sim 0.61$. Consequently, in this region the number-density of MS stars with respect to the giants is $\sim 4$ larger than in the cluster as a whole.

Mass segregation in a star cluster scales with the relaxation time, defined as $t_{\text {relax }}=\frac{N}{8 \ln N} t_{\text {cross }}$, where $t_{\text {cross }}=R / \sigma_{\mathrm{v}}$ is the crossing time, $N$ is the (total) number of stars and $\sigma_{\mathrm{v}}$ is the velocity dispersion (Binney \& Tremaine 1987). $t_{\text {relax }}$ is the characteristic time scale in which the cluster reaches some level of kinetic energy equipartition with massive stars sinking to the core and low-mass stars being transferred to the halo. Assuming a typical $\sigma_{\mathrm{v}} \approx 3 \mathrm{~km} \mathrm{~s}^{-1}$ (Binney \& Merrifield 1998) we obtain for the whole cluster $t_{\text {relax }} \sim 600 \mathrm{Myr}$, and for the core $t_{\text {relax }} \sim 6 \mathrm{Myr}$. Consequently, the presence of mass segregation and thus MF slope flattening, particularly in the core, is consistent with both estimates of $t_{\text {relax }}$ which are smaller than the age of NGC 2477.

\subsection{The moderately young open cluster NGC 2516}

The analysis of NGC 2516 followed the same steps as that of NGC 2477, noting that in the case of NGC 2516 the WEBDA 


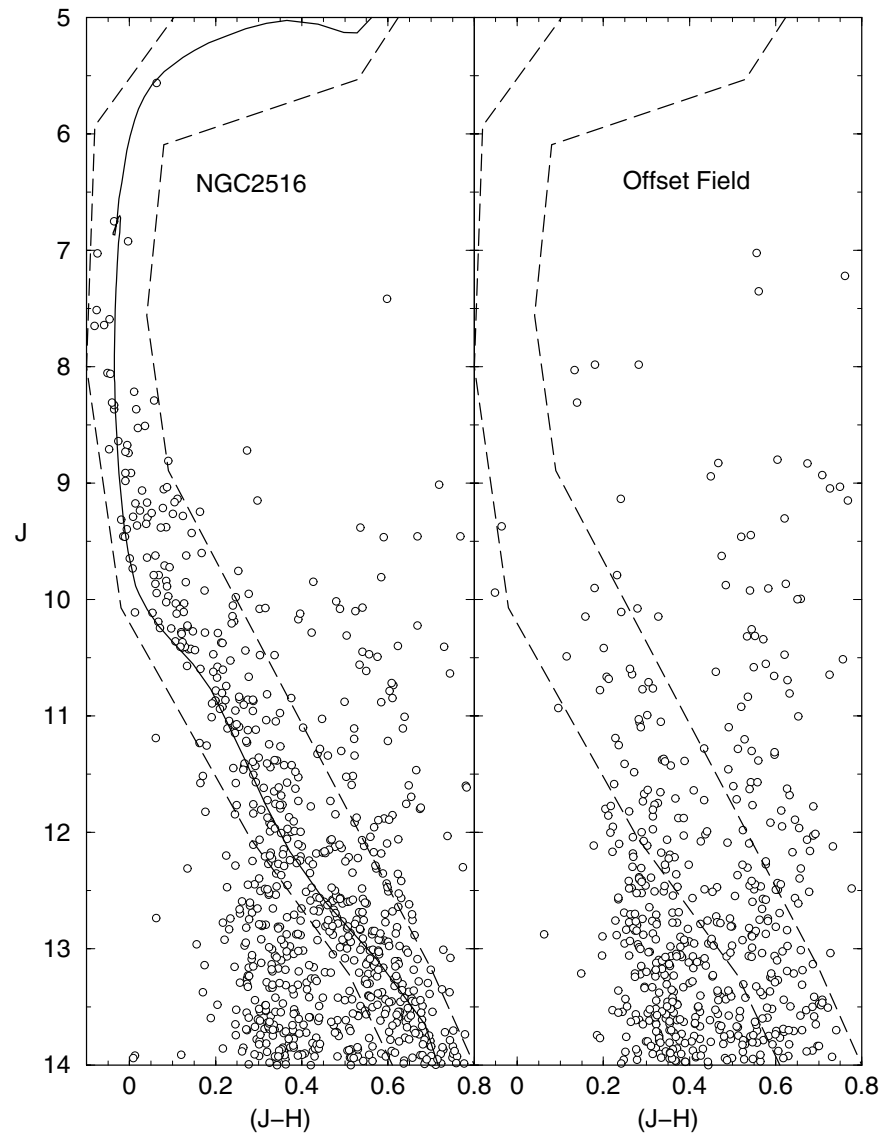

Fig. 4. Left panel: $J \times(J-H)$ CMD of the central $20^{\prime}(\sim 2.5 \mathrm{pc})$ of NGC 2516 with the best-fit Padova isochrone (age $\sim 160 \mathrm{Myr}$ ) superimposed. Right panel: CMD of the corresponding (same area) offset field. The colour-magnitude filter is shown in both panels. Note that the MS of NGC 2516 is less populated than that of NGC 2477 in Fig. 1.

coordinates (Table 1) corresponded to the actual cluster center. The Galactic coordinates of NGC 2516 are $\ell=273.82^{\circ}$ and $b=-15.86^{\circ}$. Because of the large projected area of NGC 2516 we used a radius of $r=80^{\prime}$ to extract the 2MASS photometry. The $J \times(J-H)$ CMD of the central $\left(r=20^{\prime} \sim 2.5 \mathrm{pc}\right)$ region of NGC 2516 is shown in the left panel of Fig. 4, and the corresponding, same area (extracted from 77.46' $\leq r \leq 80^{\prime}$ ) offset field is in the right panel. The young age of NGC 2516 is apparent in the CMD morphology, particularly the extended, nearly vertical MS and absence of evolved stars.

\subsubsection{Structural parameters}

The best-fit to the CMD of NGC 2516 was obtained with the 160 Myr Padova isochrone, a colour excess $E(J-H)=0.0$ $(E(B-V)=0.0)$, a distance modulus $(m-M)_{J}=8.2 \pm 0.1$, and a distance from the Sun $d_{\odot}=0.44 \pm 0.02 \mathrm{kpc}$. With this value the Galactocentric distance of NGC 2516 turns out to be $d_{\mathrm{GC}}=8.0 \pm 0.1 \mathrm{kpc}$. Considering the uncertainties associated to the isochrone fit the age of NGC 2516 gets constrained to the range $160 \pm 10 \mathrm{Myr}$. This value agrees with that estimated by Jeffries et al. (2001).

The radial density profile of stars in NGC 2516 (after applying the colour-magnitude filter - Fig. 4) is shown in Fig. 5,

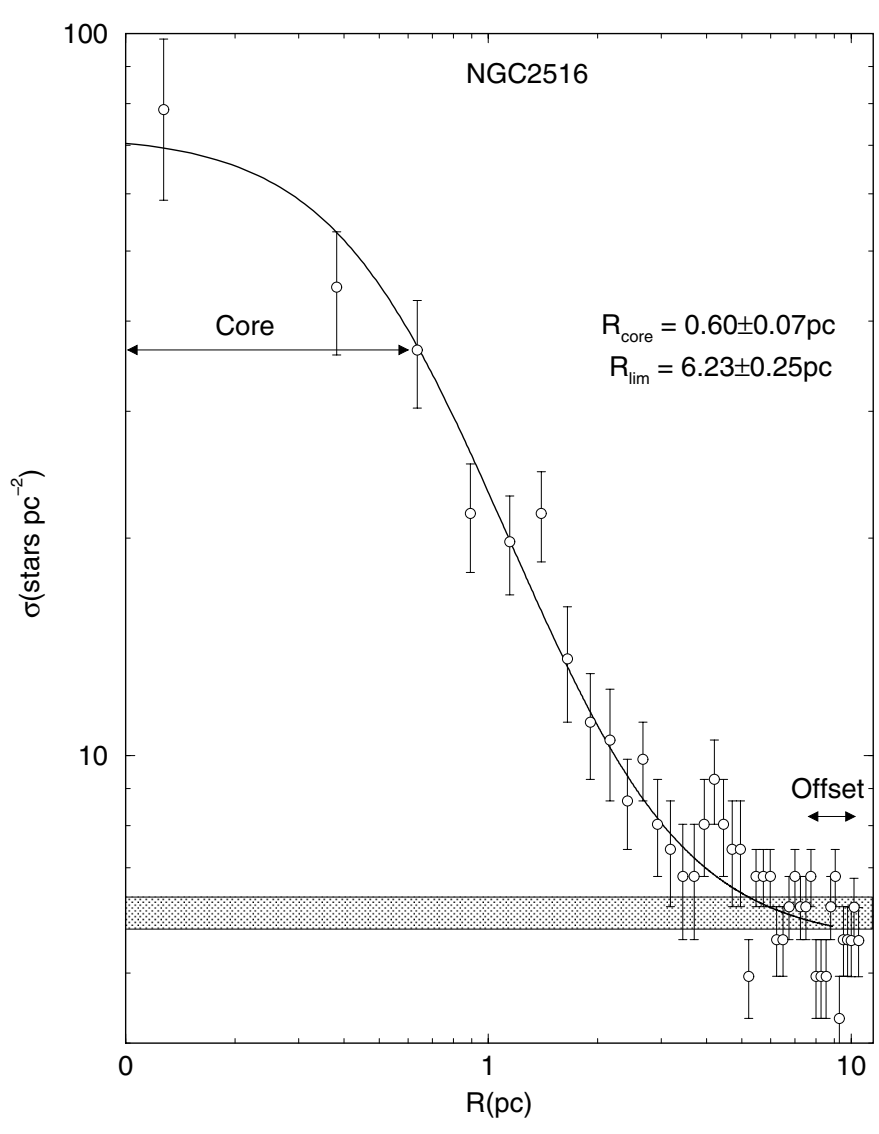

Fig. 5. Same as Fig. 2 for the radial density profile of NGC 2516.

where the background contribution corresponds to the average density of stars in the region $7.6 \leq r(\mathrm{pc}) \leq 10.18$. Similarly to NGC 2477 we restricted the radial density analysis to filtered stars with $J \leq 13.0$, corresponding to $M_{J} \leq 4.8$ and mass $m \geq 0.8 M_{\odot}$ (Sect. 3.2.2). At $J=13.0$ the photometric uncertainties amount to $\epsilon_{J} \approx 0.03$ (Bonatto et al. 2005). The fit with the two-parameter King (1966a) surface density profile to the background-subtracted radial density profile resulted in $R_{\text {core }}=0.6 \pm 0.1 \mathrm{pc}$ and a projected central density of

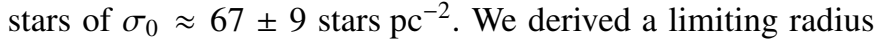
of $R_{\lim }=6.2 \pm 0.2 \mathrm{pc}$, a value $\sim 3$ times larger than the tidal radius estimated by Bergond et al. (2001) and Tadross et al. (2002). We note that in the present work the tidal radius could not be calculated since the nonlinear least-squares routine did not reach convergence with the three-parameter King (1962) model applied to the background-subtracted radial distribution of stars in NGC 2516 (Fig. 5).

\subsubsection{Mass functions}

Similarly to NGC 2477 we derive MFs for the following internal regions of NGC 2516: (i) $0.0 \leq r$ (pc) $\leq 0.6$ (core), (ii) $0.6 \leq r$ (pc) $\leq 1.7$, (iii) $1.7 \leq r$ (pc) $\leq 6.2$ and (iv) $0.0 \leq$ $r(\mathrm{pc}) \leq 6.2$ (overall). The colour-magnitude filter (Fig. 4) has been applied to the CMDs of the above regions and offset field $(7.6 \leq r(\mathrm{pc}) \leq 10.18)$ to take into account the Galactic contamination. The resulting MFs covering the mass range $0.39 \leq m\left(M_{\odot}\right) \leq 4.17$ are shown in Fig. 6 . 


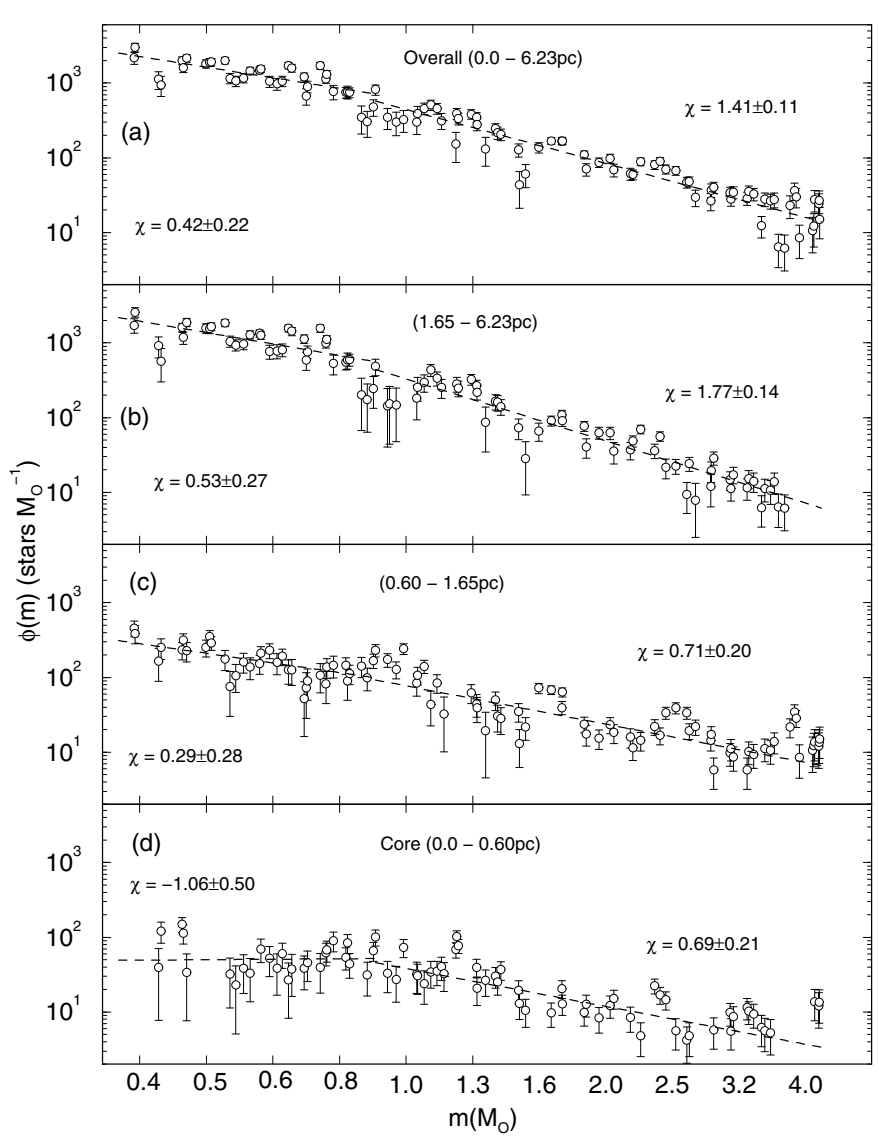

Fig. 6. Mass functions in different spatial regions of NGC 2516. Each panel contains MFs derived independently from the $J, H$ and $K_{\mathrm{S}}$ 2MASS photometry. A break in the MFs at $m_{\text {break }} \approx 0.9 M_{\odot}$ can be seen particularly in the core region (panel d)). MF fits $\left(\phi(m) \propto m^{-(1+\chi)}\right)$ are shown as dashed lines.

Parameters derived from the MFs, such as observed and extrapolated mass, projected and mass densities in each region of NGC 2516 are given in Table 2. Similarly to NGC 2477 a break in the MFs can be seen, particularly in the core region at $m=0.9 M_{\odot}$. In addition, mass-segregation effects are reflected on the slope variation with distance to the center of NGC 2516. In the mass range $0.90 \leq m\left(M_{\odot}\right) \leq 4.17$ the MF slope is similar $(\chi \approx 0.7)$ both for the core and the region $0.6 \leq r(\mathrm{pc}) \leq 1.7$, increasing to $\chi \approx 1.8$ for $1.7 \leq r(\mathrm{pc}) \leq 6.2$. In this mass range the overall MF is similar to a standard Salpeter law. In the range $0.39 \leq m\left(M_{\odot}\right) \leq 0.90$ the core MF is very flat $(\chi \approx-1.1)$ while in the other regions the slope is similar to that of the overall $\mathrm{MF}$ $(\chi \approx 0.4)$.

The sharp flattening in the MFs of NGC 2516 was previously detected by Jeffries et al. (2001) based on CCD photometry of the spatial region internal to $r \approx 31^{\prime}(\approx 4 \mathrm{pc})$. In the range $0.3 \leq m\left(M_{\odot}\right) \leq 0.7$ they derive MF slopes of $\chi=-0.75 \pm$ 0.20 or $\chi=-1.00 \pm 0.18$, depending on the (solar metallicity) isochrone models used. In the range $0.7 \leq m\left(M_{\odot}\right) \leq 3.0$ they derive slopes $\chi=1.47 \pm 0.11$ or $\chi=1.58 \pm 0.11$, for the same models as above. Considering the slope uncertainties and differences in the spatial regions sampled, the MF slopes derived in the present work are consistent with those of Jeffries et al. (2001), in both mass ranges. In addition, the total mass derived in the present work $m_{\text {tot }}=1280 \pm 162 M_{\odot}$ agrees with the value estimated by Jeffries et al. (2001), $m \approx 1240-1560 M_{\odot}$ (corrected for mass segregation for stars with $m \geq 0.3 M_{\odot}$ ). We note that previous works gave exceedingly low values for the stellar mass in NGC 2516, e.g. Bergond et al. (2001) estimate $m_{\text {tot }} \approx 170 M_{\odot}$ (for stars with $m \geq 1.1 M_{\odot}$ ), while in Tadross et al. (2002), $m_{\mathrm{tot}} \approx 36 M_{\odot}$. Extrapolated and observed core mass agree, while the extrapolated overall mass is $\sim 40 \%$ larger than the mass locked up in the observed stars.

Mass segregation in NGC 2516, as implied by the spatial variation of the MF slopes (Fig. 6), is consistent with the overall $t_{\text {relax }} \sim 90$ Myr and $t_{\text {relax }} \sim 0.4 \mathrm{Myr}$ in the core, both estimates are smaller than the age of the cluster.

\subsection{Comparing dynamical states}

The less-advanced dynamical state of NGC 2516 as compared to that of NGC 2477 is reflected both in the absolute value of the MF slopes and in the spatial rate of change of $\chi$. In the mass range $0.90 \leq m\left(M_{\odot}\right) \leq 4.17$ of $\mathrm{NGC} 2516$ the MF slope varies from $\chi \approx 0.7$ in the core to $\chi \approx 1.8$ in the region $1.7 \leq r(\mathrm{pc}) \leq 6.2$, while in the equivalent mass range $1.08 \leq m\left(M_{\odot}\right) \leq 1.95$ of NGC 2477 the slope varies from the flatter $\chi \approx 0.0$ in the core to the steeper $\chi \approx 3.3$ in the region at $5.2 \leq r(\mathrm{pc}) \leq 11.6$. Thus, in this mass range the radial variation of the MF slope in NGC 2516 is $\frac{\Delta \chi}{\Delta r}=(0.30 \pm 0.19) \mathrm{pc}^{-1}$, while in NGC $2477 \frac{\Delta \chi}{\Delta r}=(0.43 \pm 0.19) \mathrm{pc}^{-1}$. A similar situation with respect to MF slope values and radial variations occurs in the mass range corresponding to the MF breaks in NGC 2516 with $\frac{\Delta \chi}{\Delta r}=(0.4 \pm 0.3) \mathrm{pc}^{-1}$ and NC 2477 with $\frac{\Delta \chi}{\Delta r}=(0.5 \pm 0.2) \mathrm{pc}^{-1}$. Finally, the overall MF slopes in NGC 2516 and NGC 2477 are essentially the same in the low-mass MS $(\chi \approx 0.4)$ and in the high-mass $\operatorname{MS}(\chi \approx 1.4)$.

Thus, the discussions above show that the fact that NGC 2477 is $\sim 7$ times as old as NGC 2516 turns out to be more important to the internal dynamical evolution than NGC 2477 being $\sim 4$ times more massive than NGC 2516.

\section{Multi-parametric analysis}

The analysis method described in Sects. 3.1 and 3.2 respectively for NGC 2477 and NGC 2516 was applied to the remaining clusters in Table 1. We derived a homogeneous set of parameters associated to the internal structure (core and limiting radii), stellar evolution (extrapolated mass) and dynamical evolution (core and overall mass and mass density, and MF slope). We do not include in the statistical analyses below the tidal radius, since convergence in the nonlinear least-squares routine with the three-parameter King (1962) model has been reached only for the background-subtracted radial profiles of $\mathrm{M} 26\left(R_{\mathrm{tidal}}=15 \pm 7 \mathrm{pc} \sim 1.9 \times R_{\text {lim }}\right), \operatorname{NGC} 2477\left(R_{\text {tidal }}=16 \pm\right.$ $\left.2 \mathrm{pc} \sim 1.4 \times R_{\text {lim }}\right)$, M $67\left(R_{\text {tidal }}=16 \pm 3 \mathrm{pc} \sim 1.4 \times R_{\text {lim }}\right)$ and NGC $188\left(R_{\text {tidal }}=22 \pm 3\right.$ pc $\left.\sim 1.8 \times R_{\text {lim }}\right)$. As a comparison between conceptually different estimates of cluster dimension, in these four open clusters the ratio $R_{\text {tidal }} / R_{\text {lim }}$ varies from 1.4 to 1.9. Thus, for sample completeness purposes we use the limiting radius in what follows. 
Table 3. Structural and dynamical-related parameters of the target open clusters.

\begin{tabular}{|c|c|c|c|c|c|c|c|c|c|c|c|c|c|c|}
\hline \multirow[b]{2}{*}{ Cluster } & \multicolumn{6}{|c|}{ Core } & \multicolumn{6}{|c|}{ Overall } & \multirow{3}{*}{$\begin{array}{c}m_{\text {break }} \\
\left(M_{\odot}\right) \\
(14)\end{array}$} & \multirow[b]{2}{*}{$c^{*}$} \\
\hline & $\begin{array}{l}R_{\text {core }} \\
(\mathrm{pc})\end{array}$ & $\begin{array}{c}m_{\mathrm{obs}} \\
\left(10^{2} M_{\odot}\right)\end{array}$ & $\begin{array}{c}m_{\text {tot }} \\
\left(10^{2} M_{\odot}\right)\end{array}$ & $\sigma$ & $\rho$ & $x$ & $\begin{array}{l}R_{\lim } \\
(\mathrm{pc})\end{array}$ & $\begin{array}{c}m_{\mathrm{obs}} \\
\left(10^{2} M_{\odot}\right)\end{array}$ & $\begin{array}{c}m_{\text {tot }} \\
\left(10^{2} M_{\odot}\right)\end{array}$ & $\sigma$ & $\rho$ & $x$ & & \\
\hline (1) & $(2)$ & (3) & (4) & (5) & (6) & (7) & $(8)$ & (9) & (10) & (11) & (12) & (13) & & $(15)$ \\
\hline M 26 & $0.8 \pm 0.1$ & $1.2 \pm 0.3$ & $2.9 \pm 0.9$ & $150 \pm 48$ & $142 \pm 46$ & $1.3 \pm 0.2$ & $8.0 \pm 0.5$ & $6.8 \pm 1.6$ & $19 \pm 7$ & $9.4 \pm 3.3$ & $0.9 \pm 0.3$ & $1.5 \pm 0.2$ & $(\dagger)$ & 1.0 \\
\hline NGC 2516 & $0.6 \pm 0.1$ & $0.9 \pm 0.1$ & $0.9 \pm 0.2$ & $82 \pm 14$ & $103 \pm 17$ & $0.7 \pm 0.2$ & $6.2 \pm 0.2$ & $9.1 \pm 0.8$ & $13 \pm 2$ & $10.5 \pm 1.3$ & $1.3 \pm 0.2$ & $1.4 \pm 0.1$ & 0.90 & 1.0 \\
\hline NGC 2287 & $1.1 \pm 0.1$ & $0.8 \pm 0.2$ & $0.9 \pm 0.2$ & $22 \pm 5$ & $15 \pm 4$ & $0.2 \pm 0.3$ & $7.0 \pm 0.7$ & $5.7 \pm 0.7$ & $7.2 \pm 0.9$ & $4.7 \pm 0.6$ & $0.5 \pm 0.1$ & $1.5 \pm 0.2$ & 1.01 & 0.8 \\
\hline M 48 & $0.9 \pm 0.2$ & $0.5 \pm 0.1$ & $0.6 \pm 0.1$ & $26 \pm 3$ & $23 \pm 3$ & $0.3 \pm 0.2$ & $8.8 \pm 0.4$ & $4.8 \pm 0.7$ & $6.3 \pm 1.5$ & $2.6 \pm 0.6$ & $0.22 \pm 0.05$ & $1.3 \pm 0.2$ & 1.06 & 1.0 \\
\hline M 93 & $0.7 \pm 0.1$ & $1.1 \pm 0.1$ & $1.4 \pm 0.1$ & $85 \pm 8$ & $88 \pm 9$ & $-0.2 \pm 0.2$ & $7.2 \pm 0.6$ & $7.4 \pm 0.6$ & $17 \pm 5$ & $10.2 \pm 3.4$ & $1.0 \pm 0.3$ & $1.2 \pm 0.1$ & - & 1.0 \\
\hline NGC 5822 & $1.1 \pm 0.1$ & $1.1 \pm 0.1$ & $1.6 \pm 0.4$ & $43 \pm 11$ & $30 \pm 7$ & $0.6 \pm 0.2$ & $8.0 \pm 0.4$ & $13.6 \pm 1.0$ & $26 \pm 10$ & $13 \pm 5$ & $1.2 \pm 0.4$ & $1.7 \pm 0.1$ & - & 0.9 \\
\hline NGC 2477 & $1.4 \pm 0.1$ & $4.1 \pm 0.4$ & $5.2 \pm 0.8$ & $85 \pm 13$ & $45 \pm 7$ & $0.0 \pm 0.2$ & $11.6 \pm 0.7$ & $27 \pm 14$ & $53 \pm 16$ & $13 \pm 4$ & $0.8 \pm 0.2$ & $1.5 \pm 0.1$ & 1.08 & 0.9 \\
\hline NGC 3680 & $0.5 \pm 0.2$ & $0.3 \pm 0.1$ & $0.4 \pm 0.1$ & $45 \pm 6$ & $64 \pm 9$ & $-1.1 \pm 0.4$ & $5.8 \pm 0.6$ & $1.7 \pm 0.2$ & $3.6 \pm 1.1$ & $3.4 \pm 1.0$ & $0.5 \pm 0.1$ & $0.5 \pm 0.3$ & - & 1.0 \\
\hline IC 4651 & $0.8 \pm 0.1$ & $0.9 \pm 0.1$ & $1.0 \pm 0.1$ & $54 \pm 6$ & $53 \pm 6$ & $-1.4 \pm 0.3$ & $6.0 \pm 0.3$ & $7.3 \pm 0.7$ & $8.4 \pm 1.0$ & $7.5 \pm 0.9$ & $0.9 \pm 0.1$ & $0.6 \pm 0.3$ & 0.97 & 0.9 \\
\hline M 67 & $1.1 \pm 0.1$ & $1.3 \pm 0.2$ & $1.4 \pm 0.2$ & $34 \pm 4$ & $22 \pm 3$ & $-2.5 \pm 0.4$ & $11.7 \pm 0.6$ & $9.4 \pm 0.7$ & $9.9 \pm 1.2$ & $2.5 \pm 0.3$ & $0.16 \pm 0.02$ & $-0.3 \pm 0.2$ & 0.80 & 1.0 \\
\hline NGC 188 & $1.3 \pm 0.1$ & $0.7 \pm 0.1$ & $6.1 \pm 0.3$ & $99 \pm 42$ & $53 \pm 23$ & $0.6 \pm 0.7$ & $11.9 \pm 0.5$ & $3.8 \pm 0.1$ & $38 \pm 16$ & $8.5 \pm 3.6$ & $0.54 \pm 0.23$ & $1.9 \pm 0.7$ & $(\ddagger)$ & 1.0 \\
\hline
\end{tabular}

Notes. $m_{\text {obs }}$ and $m_{\text {tot }}$ in Cols. 3, 4, 9 and 10 includes the mass in the evolved stars mass. $m_{\text {break }}$ in Col. 14 is the mass where the MF break begins. $\chi$ in Cols. 7 and 13 is the MF slope in the mass range $m \geq m_{\text {break }}$. $\dagger$ - because of the distance from the Sun, the low-mass end of the MF of M 26 occurs at $m=0.98 M_{\odot}$. $(\ddagger)$ - because of the distance from the Sun and old age the MF of NGC 188 is restricted to the mass range $0.9 M_{\odot}-1.0 M_{\odot}$. The uncertainty in $m_{\text {break }}$ in Col. 14 is $\approx 0.05 M_{\odot}$ in all clusters. The uncertainty in $c^{*}$ in Col. 15 is 0.1 in all clusters except for NGC 3680 for which it is 0.2 . Units of $\sigma$ (Cols. 5 and 11 ) and $\rho$ (Cols. 6 and 12) are $M_{\odot} \mathrm{pc}^{-2}$ and $M_{\odot} \mathrm{pc}^{-3}$, respectively.

Except for cluster age and Galactocentric distance which are listed in Table 1, the remaining parameters are listed in Table 3, in which we consider separately the core and overall regions. By columns: (1) - cluster designation; (2) - core radius; (3) - observed core mass, which includes the mass of the evolved stars down to the mass corresponding to the 2MASS faint magnitude limits (Sect. 3.1.3); (4) - extrapolated core mass, including stars with $m=0.08 M_{\odot} ;(5)$ - projected core mass density $\left(m / \pi R_{\text {core }}^{2}\right)$; $(6)$ - core mass density $\left(m / \frac{4}{3} \pi R_{\text {core }}^{3}\right)$; (7) - core MF slope (for the mass range $m \geq m_{\text {break }}$ ); (8) limiting radius; (9) - observed overall mass; (10) - extrapolated overall mass; (11) - overall projected mass density; and (12) - overall mass density. The overall MF slope ( $\left.m \geq m_{\text {break }}\right)$ is given in Col. 13. In Col. 14 we give $m_{\text {break }}$, the stellar mass where the MF break occurs. Finally, in Col. 15 we give $c^{*}=\log \left(R_{\lim } / R_{\text {core }}\right)$, which can be measured essentially in all open clusters. Derived from the limiting radius, $c^{*}$ is not the same as the concentration parameter of globular clusters, which is based on the tidal radius (Trager et al. 1995). Since the tidal radius can only be measured for the most populous open clusters and/or those at high Galactic latitudes (see above), we emphasize the importance of using in the future $c^{*}$ for statistical purposes when dealing with samples of open clusters. The relatively narrow range of $c^{*}$ from $\approx 0.8$ to $\approx 1.0$ is characteristic of loose star clusters (Trager et al. 1995).

To characterize the sample we present average values of some parameters. For the massive $\left(m \geq 1000 M_{\odot}\right)$ clusters we obtain $\left\langle R_{\text {core }}\right\rangle=1.0 \pm 0.3 \mathrm{pc},\left\langle M_{\text {core }}\right\rangle=(3 \pm 2) \times 10^{2} M_{\odot}$, $\left\langle\chi_{\text {core }}\right\rangle=0.5 \pm 0.5,\left\langle R_{\lim }\right\rangle=8.8 \pm 2.3 \mathrm{pc},\left\langle M_{\text {overall }}\right\rangle=$ $(2.8 \pm 1.5) \times 10^{3} M_{\odot}$ and $\left\langle\chi_{\text {overall }}\right\rangle=1.5 \pm 0.2$. For the lessmassive clusters they are $\left\langle R_{\text {core }}\right\rangle=0.9 \pm 0.2 \mathrm{pc},\left\langle M_{\text {core }}\right\rangle=$ $(0.8 \pm 0.4) \times 10^{2} M_{\odot},\left\langle\chi_{\text {core }}\right\rangle=-0.9 \pm 1.1,\left\langle R_{\text {lim }}\right\rangle=7.9 \pm$ $2.5 \mathrm{pc},\left\langle M_{\text {overall }}\right\rangle=(0.7 \pm 0.2) \times 10^{3} M_{\odot}$ and $\left\langle\chi_{\text {overall }}\right\rangle=0.7 \pm$ 0.7. On average the core and overall MFs of the less-massive clusters tend to be flatter than those of the massive ones. Core and overall masses of the massive clusters are $\sim 4$ times larger than those of the less-massive ones. Within the standard deviations core and limiting radii are similar on average, in both types of clusters.

Because of the reduced number of clusters in this work, the results below should be taken as a sampling of the class of open clusters. On the other hand, the statistical significance and homogeneous method from which the parameters were derived guarantee that the results may serve as indicative of trends and correlations.

\subsection{Cluster structural and evolutionary parameters vs. cluster age}

Relations involving cluster parameters and cluster age are investigated in Fig. 7 , in which we use different symbols to identify massive and less-massive clusters. Among the parameters tested the best correlation with age occurs for $R_{\lim }$ (panel a). This correlation should be expected since young (age $\leq 700 \mathrm{Myr}$, Friel 1995) clusters with large diameters probably are not bound systems (Lyngå 1982; Janes et al. 1988), and small, massive clusters will be dissolved due to the strong effects of the internal dynamics (Tadross et al. 2002). This correlation was not present in the sample studied by Tadross et al. (2002). The core radius in panel (g) does not seem to correlate with age.

An interesting pattern occurs in the plots $\rho_{\text {core }} \times$ age (panel l) and $\sigma_{\text {core }} \times$ age (panel i) which may reflect different evolutionary processes in the core of more-massive clusters with respect to less-massive ones. A similar pattern occurs in the plot $\chi_{\text {overall }} \times$ age (panel $\mathrm{d}$ ), in which massive and less-massive clusters seem to evolve differently, in the sense that old, lessmassive clusters tend to have flatter overall MFs than old massive ones. Because of their looser nature with respect to 


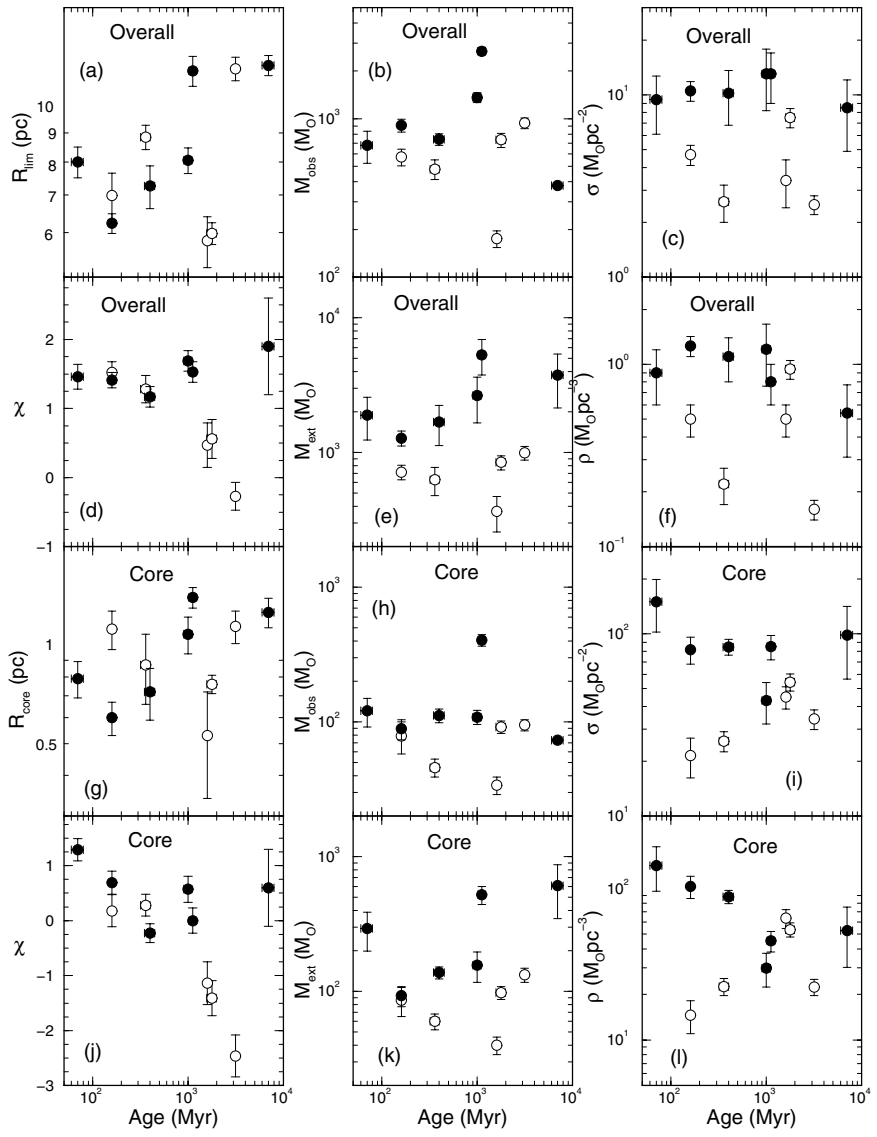

Fig. 7. Relations of cluster parameters with cluster age. Filled symbols correspond to massive clusters (overall mass $\geq 1000 M_{\odot}$ ).

massive clusters, old less-massive clusters have lost a significant fraction of the low-mass content through internal processes such as mass segregation and evaporation, and external ones such as tidal stripping, disk shocking and encounters with molecular clouds (Bergond et al. 2001). As a consequence, a higher flattening degree in the MFs of old less-massive clusters with respect to old massive ones, is expected. A similar pattern occurs with respect to the relation of the core MF slope with age in panel $\mathrm{j}$.

The projected overall mass density (panel c), total core mass (panel k) and overall mass density panel f) do not correlate with age, but massive and less-massive clusters in general occupy distinct positions in the plots.

Considering the significant scatter in the plots we find no indication of correlation with cluster age of total overall mass (panel e), observed overall mass (panel b) and observed core mass (panel h). A larger cluster sample is necessary to check these points.

\subsection{Cluster structural and evolutionary parameters vs. Galactocentric distance}

Relations involving cluster parameters and Galactocentric distance are investigated in Fig. 8. The plot in panel f suggests that there is no correlation of cluster mass with Galactocentric distance. Although the scatter in most plots is considerable,
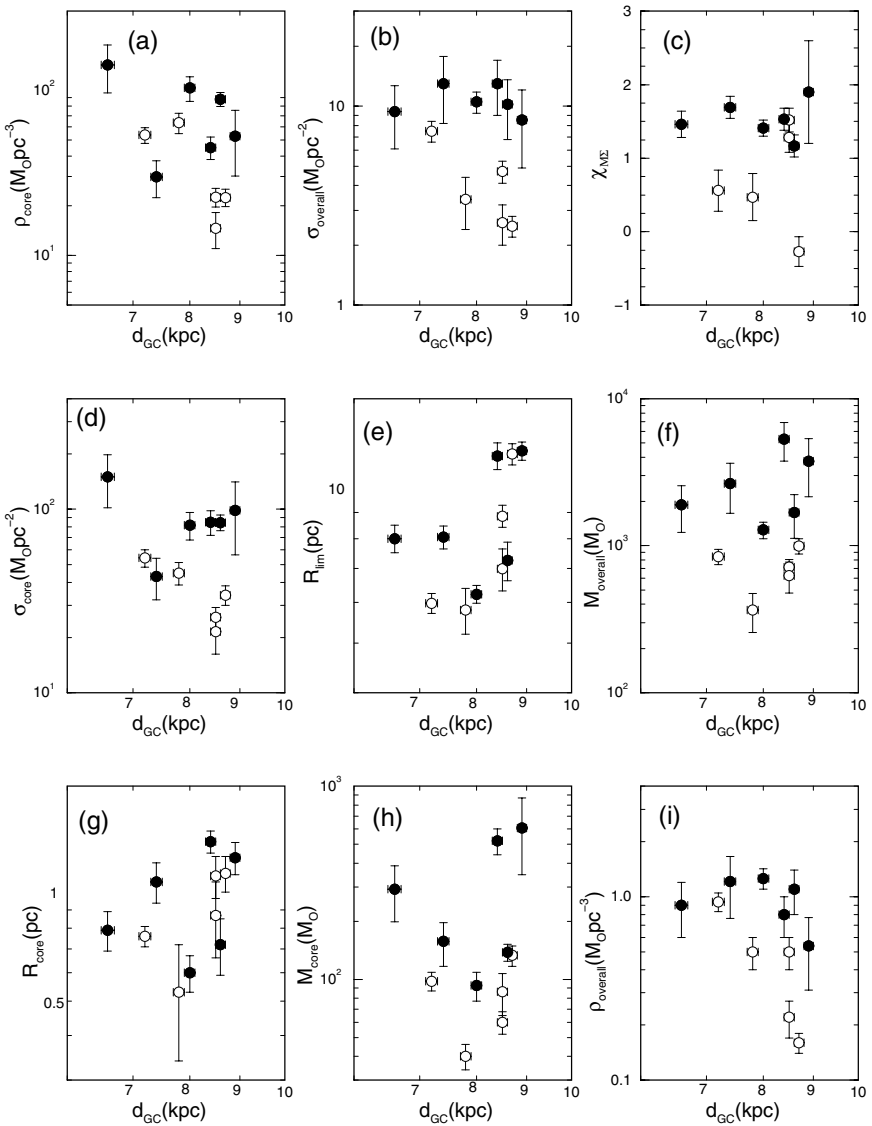

Fig. 8. Relations of cluster parameters with Galactocentric distance. Symbols as in Fig. 7.

the plot in panel e suggests a correlation of $R_{\lim }$ with Galactocentric distance $d_{\mathrm{GC}}$, similar to the results of Lyngå (1982) and Tadross et al. (2002). This correlation holds for massive and less-massive clusters. The core mass density (panel a) and, to a less extent the overall mass density (panel i), seem to fall for clusters at larger $d_{\mathrm{GC}}$, probably because of the slight dependence of core radius on $d_{\mathrm{GC}}$ (panel g). Finally, in panel i of Fig. 9 we examine the relation of $d_{\mathrm{GC}}$ with cluster age. Young and old clusters, with any mass, are similarly distributed in terms of $d_{\mathrm{GC}}$. This apparently contradicts the conclusion of Lyngå (1982) in which older clusters are found preferentially in the outer parts of the Galaxy, while younger clusters are evenly distributed. However, our result is based on 11 clusters only, which sample a limited range in $d_{\mathrm{GC}}$ in the solar surroundings.

\subsection{Core vs. overall parameters}

In Fig. 9 we examine relations involving core and overall parameters. The tight correlations involving core and limiting radii (panel g) and core and overall total mass (panel d) are probably consequences of size and mass scaling, in the sense that bigger clusters tend to have bigger cores and larger masses. Linear least-squares fits to the points resulted in correlation coefficients of 0.85 and 0.84 , respectively for the plots in panels $\mathrm{d}$ and $\mathrm{g}$. The projected overall density (panel a) and overall mass density (panel h) seem to correlate as well, although with 

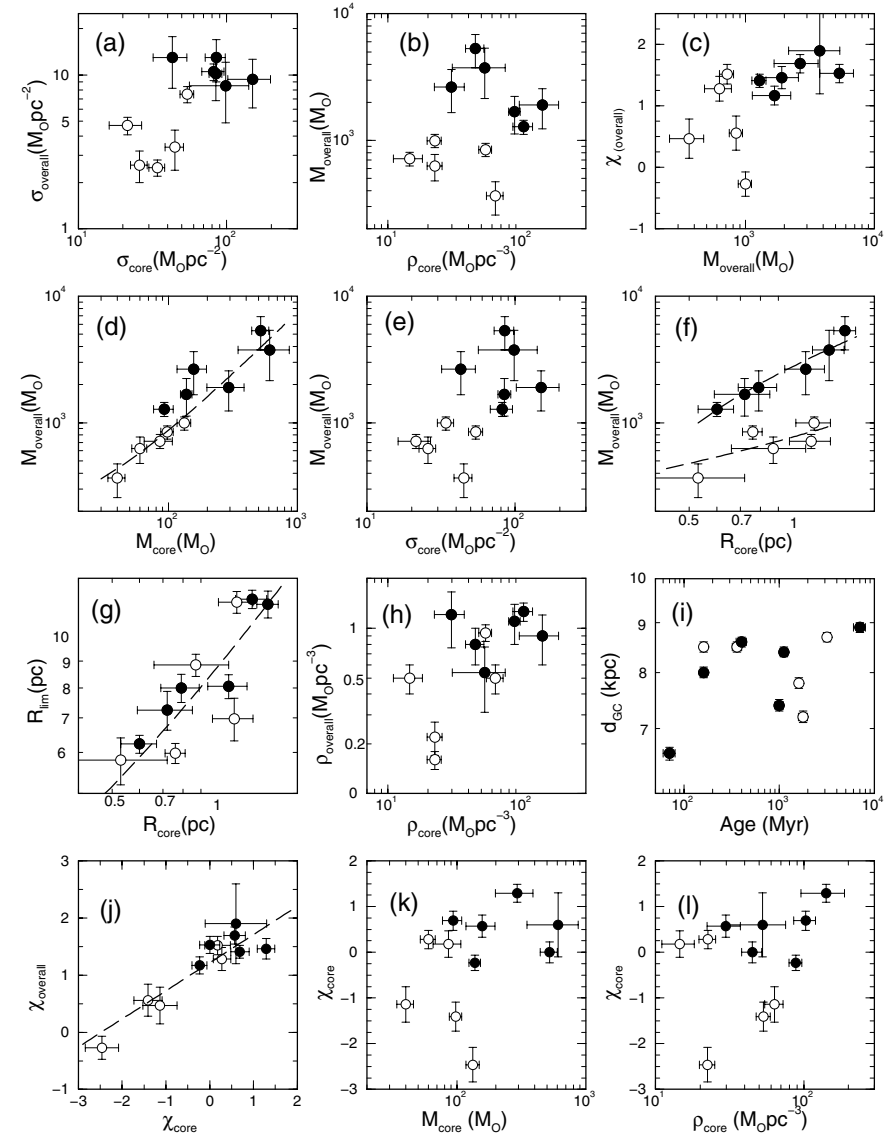

Fig. 9. Relations involving core and overall parameters. Symbols as in Fig. 7. Dashed lines correspond to linear least-squares fits to the points.

significant scatter. Core radius and overall mass are tightly correlated (panel f) as well. However, massive and less-massive clusters seem to follow separate, but nearly parallel, paths in the plot. Correlation coefficients are 0.97 and 0.68 , respectively for the fits of the massive and less-massive clusters.

Total overall mass and overall MF slope correlate (panel c). In this case the MS of massive clusters is characterized by a narrow range of MF slopes, $1 \leq \chi \leq 2$, while the MS of less-massive clusters has $\chi$ spread over a large range. No definite pattern emerges from the relations of total overall mass with core mass density (panel b) and core projected density (panel e).

Finally, the core and overall MF slopes are tightly correlated (panel j), with a correlation coefficient of 0.90 . Total core mass and core MF slope (panel k) present a similar pattern as total overall mass and overall MF slope (panel c).

\section{MF break and the universality of the IMF}

Since the early work of Salpeter (1955) in which the IMF of solar-neighbourhood stars in the range $0.4 M_{\odot}$ to $10 M_{\odot}$ was described by the function $\phi(m) \propto m^{-(1+\chi)}$, with $\chi=1.35$, evidence has been accumulating pointing to the presence of breaks in the MF of low-mass stars, in the sense that it tends to be flatter than the MF of high-mass stars. On the highmass end, the Salpeter IMF has been shown to extend up to very-massive stars, such as $m \approx 120 M_{\odot}$ (Scalo 1986; Massey 1998). However, star counts in the solar neighbourhood show that the IMF for the low-mass range breaks up in two powerlaws, $\chi=1.2 \pm 0.3$ for $0.5 \leq m\left(M_{\odot}\right) \leq 1.0$ and $\chi=0.3 \pm 0.7$ for $0.08 \leq m\left(M_{\odot}\right) \leq 0.5$. In the brown dwarf mass range the slope seems to be even flatter, $\chi \approx-0.5$ (Kroupa 2001, 2002).

Present-day data suggest that the IMF may have a universal character, in the sense that it probably is similar in very different environments, from star-forming regions in molecular clouds to rich star clusters and the field (Kroupa 2002). As additional observational evidence of similar IMFs in different environments von Hippel et al. (1996) discuss star counts in the near-IR, in the optical with Hubble Space Telescope data and from the Hubble Deep Field which indicate that field stars of the Galactic thick disk and halo have a LF similar to that of the solar neighbourhood down to $0.08 M_{\odot}$ stars - stellar populations differing significantly in local density and chemical abundances. As pointed out by Kroupa (2002) this scenario appears to indicate that the distribution of stellar masses should depend only on the process of molecular cloud fragmentation. In this case, the fragmentation would have to produce similar IMFs despite very different initial conditions, a physical process which is not yet fully understood (Kroupa 2002).

The relatively populous nature of the open clusters included in the present study offers an opportunity to investigate the presence and properties of the MF break in clusters with a high statistical significance. However, the basically solar metallicity of the present clusters does not allow inferences on relations of $m_{\text {break }}$ with metallicity (e.g. von Hippel et al. 1996) or on the possible dependence of MF flattening (in the range $m \leq 0.7 M_{\odot}$ ) with metallicity, as discussed in Kroupa (2002).

An MF slope break, followed by a MF slope flattening (turnover) for lower masses, is present in six clusters of the present sample. The MFs of NGC 2477 clearly present a break at $m_{\text {break }} \approx 1.08 M_{\odot}$ (Fig. 3), while in NGC 2516 the break occurs at $m_{\text {break }} \approx 0.90 M_{\odot}$ (Fig. 6). In both clusters the break consistently occurs in the four spatial MFs presented in Figs. 3 and 6 . In the remaining clusters of the present paper the break is present in the MFs of M 48 at $m_{\text {break }} \approx 1.06 M_{\odot}$ (overall MF), NGC 2287 at $m_{\text {break }} \approx 1.01 M_{\odot}$ (core and overall MF), IC 4651 at $m_{\text {break }} \approx 0.97 M_{\odot}$ (overall MF) and M 67 at $m_{\text {break }} \approx 0.80 M_{\odot}$ (core and overall MF). The core and overall MFs of these clusters are shown in Fig. 10 along with the respective MF fits. In Col. 2 of Table 4 we give the mass range over which we measure the MF flattening ( $m \leq m_{\text {break }}$ ), while the corresponding core and overall MF slopes in this mass range are in Cols. 3 and 4 , respectively.

The flattening degree in the MF for masses below $m_{\text {break }}$ is indeed quite high (Table 4) when compared to that in the mass range above $m_{\text {break }}$ (Table 3), particularly in the core. Mass segregation throughout the cluster and low-mass star loss through dynamical evaporation certainly contribute to MF flattening. This effect is expected to be more significant in the old and less-massive clusters (Bergond et al. 2001). In the mass range $m \geq m_{\text {break }}$ this effect is confirmed by the relations $\chi \times$ age both in the core and overall MFs (Fig. 7, panels $j$ and d, respectively). In addition, observational consequences of the presence of a significant fraction of undetected binaries (and 


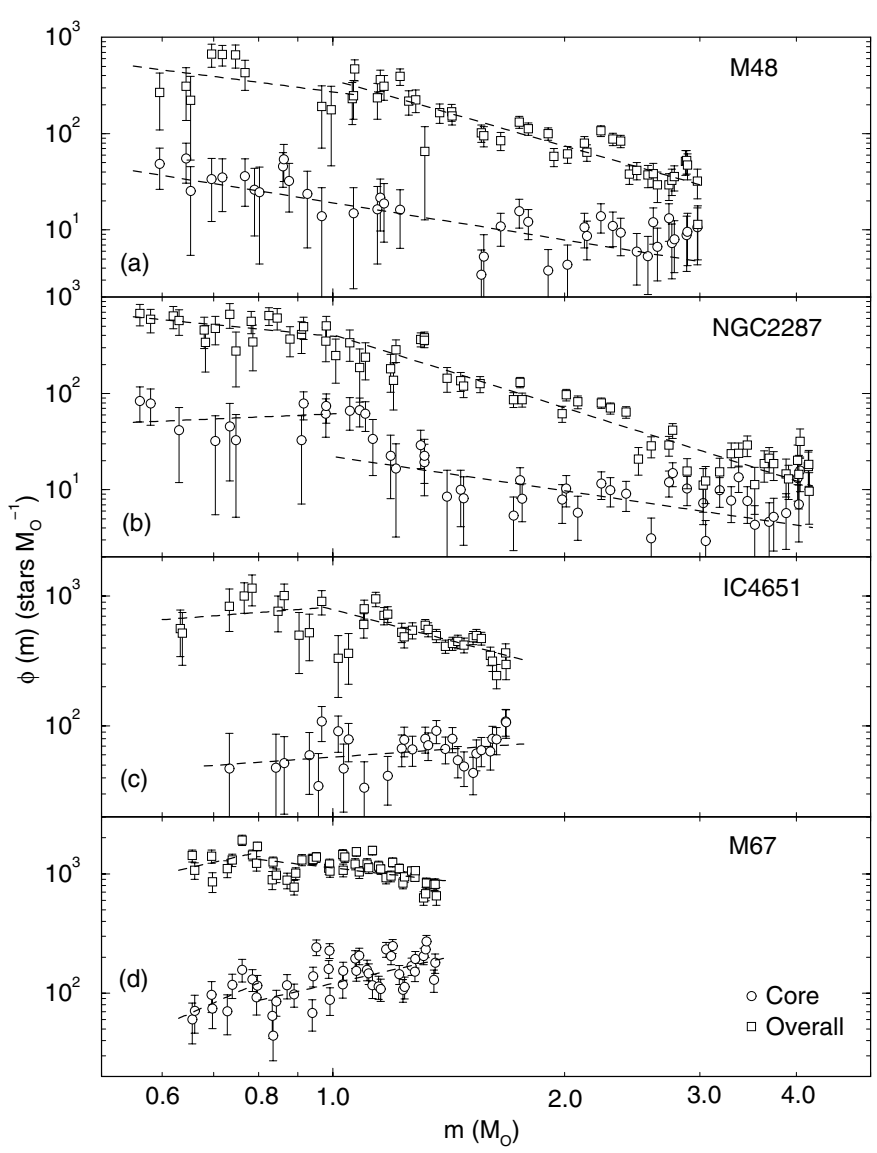

Fig. 10. Open clusters with a MF break in the low-mass MS. Because of the significant error bars in the MF of NGC 2287 (panel b)) the low-mass MS and high-mass MS fits do not match at $m \approx 1 M_{\odot}$. Core and overall regions are considered separately.

Table 4. Core and overall MF slopes for $m \leq m_{\text {break }}$.

\begin{tabular}{lccc}
\hline \hline Cluster & $\begin{array}{c}\Delta m \\
\left(M_{\odot}\right)\end{array}$ & $\chi$ (core) & $\chi$ (overall) \\
& $(2)$ & $(3)$ & $(4)$ \\
\hline NGC 2516 & $0.40-0.90$ & $-1.1 \pm 0.5$ & $+0.4 \pm 0.2$ \\
NGC 2287 & $0.56-1.01$ & $-1.3 \pm 0.5$ & $-0.2 \pm 0.3$ \\
M 48 & $0.60-1.06$ & $+0.3 \pm 0.2$ & $0.0 \pm 0.7$ \\
NGC 2477 & $0.76-1.08$ & $-2.3 \pm 0.5$ & $+0.4 \pm 0.4$ \\
IC 4651 & $0.63-0.97$ & $-1.4 \pm 0.3$ & $-1.4 \pm 0.7$ \\
M 67 & $0.66-0.80$ & $-3.9 \pm 1.1$ & $-2.5 \pm 0.2$ \\
\hline
\end{tabular}

Notes. Column 2: mass range where the MF flattening is observed. Columns 3 and 4: mass-function slope $\left(\phi(m) \propto m^{-(1+\chi)}\right)$ for $m \leq$ $m_{\text {break. }}$.

higher-order multiple systems) in a cluster are, among others, the underestimation of the number of low-mass with respect to higher-mass stars and widening of the MS. Consequently, somewhat flat MFs are expected to occur in clusters with a high fraction of unresolved binaries (e.g. Bonatto et al. 2005 and references therein). Indeed, based on proper-motion considerations Bica \& Bonatto (2005) derived that the fraction of undetected binaries in the cores of M 26, NGC 2287, M 48, M 93,
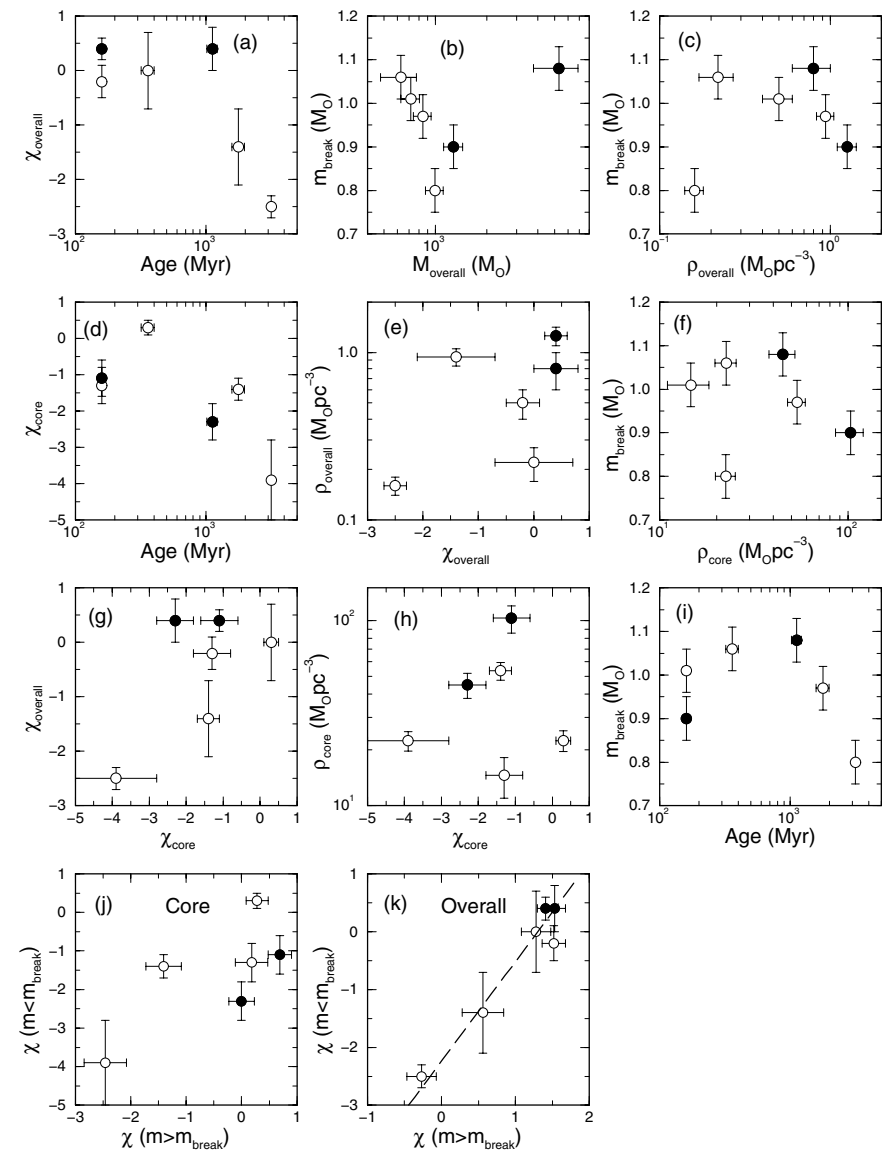

Fig. 11. Relations involving parameters of the clusters with the MF break. $\chi_{\text {core }}$ and $\chi_{\text {overall }}$ in panels a), d), e), g) and h) are the MF slopes derived for the range $m \leq m_{\text {break }}$. Symbols asin Fig. 7 .

NGC 5822, NGC 3680, IC 4651 and M 67 is in the range $15 \%$ to $50 \%$. In the core of the old open cluster NGC 188 Bonatto et al. (2005) estimated that the fraction of undetected binaries is probably around $100 \%$. However, even fractions of $\sim 100 \%$ of unresolved binaries cannot produce such flat MFs as those detected above (Bonatto et al. 2005). Thus, the flat MFs and the presence of the MF break in clusters of any age and mass (Table 3 and Fig. 10) must essentially reflect the effects of the internal dynamics of clusters on the MFs and/or some fundamental property of the IMF associated to different conditions in star formation. Kroupa (2002) points out that systematic differences in the IMF should appear between low-density environments and high-density regions. To investigate this issue we test relations involving parameters of the clusters which present the MF break. The results are in Fig. 11.

Systematic MF flattening with time due to dynamical evolution is manifest in panel a of Fig. 11 which shows that the overall MF slope (in the mass range $m \leq m_{\text {break }}$ ) decreases with age, particularly for the less-massive clusters. A similar trend is also present in the core MF slope (panel d). The decrease of core and overall MF slopes with age was present in the mass range $m \geq m_{\text {break }}$ (Fig. 7, panels j and d).

Similarly to the mass range $m \geq m_{\text {break }}$, core and overall MF slopes correlate as well (panel g), particularly in the less-massive clusters. The scatter hinders conclusions on 


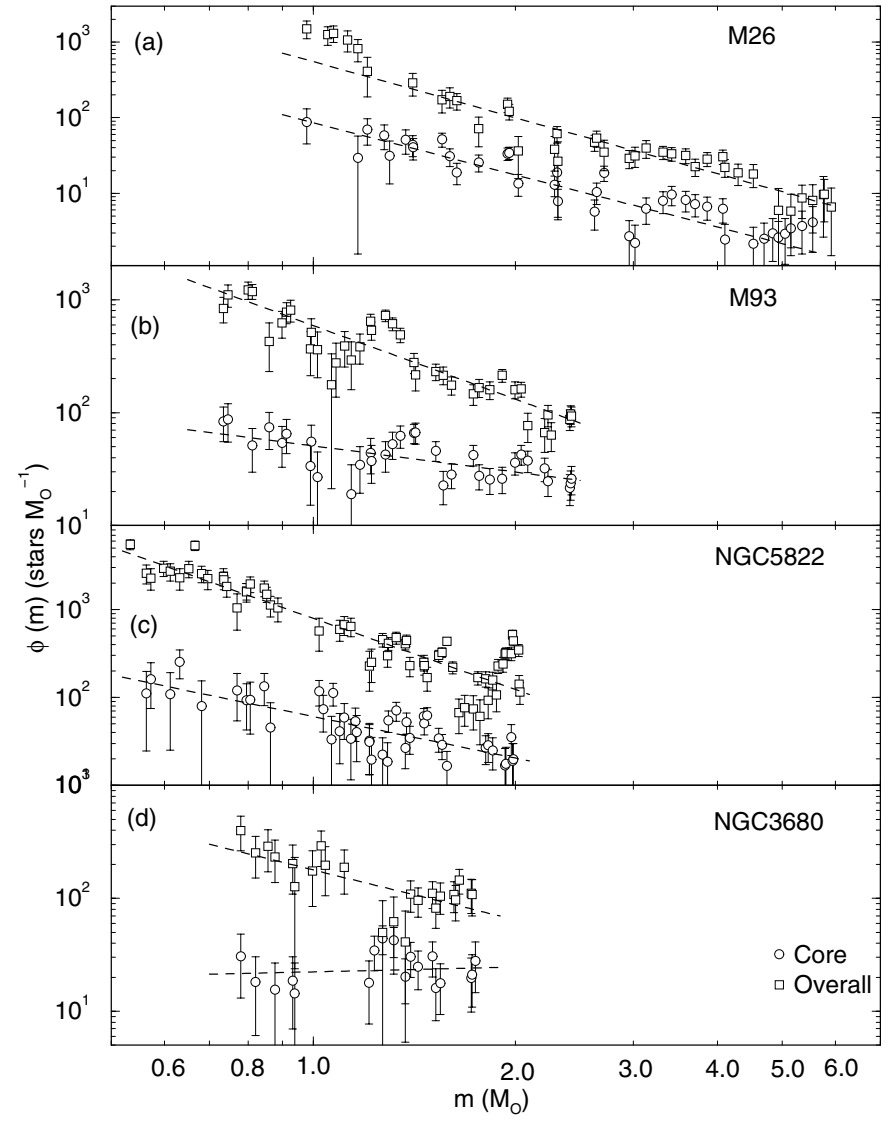

Fig. 12. Open clusters with no clear evidence of a MF break. Because of the distance from the Sun, the MF of M 26 in panel a) reaches down to $m \approx 0.98 M_{\odot}$.

dependences of MF slope on mass density in the core (panel h) and in the whole cluster (panel e). Except for the populous cluster NGC 2477, $m_{\text {break }}$ seems to decrease with overall cluster mass (panel b). The same trend occurs for core mass, since core and overall mass are tightly correlated (panel j in Fig. 9). Within the scatter we do not find correlations of $m_{\text {break }}$ with mass density, both in the core (panel f) and in the whole cluster (panel c). However, $m_{\text {break }}$ seems to decrease with cluster age for the clusters older than 1 Gyr (panel i).

MF slopes in the mass range $m \leq m_{\text {break }}$ (break region) and $m \geq m_{\text {break }}$ correlate, both in the core (panel j) and particularly in the whole cluster (panel $\mathrm{k}$, correlation coefficient 0.96 ). This suggests a common origin for the mechanisms responsible for the excess MF flattening in $m \leq m_{\text {break }}$ and that in $m \geq m_{\text {break }}$.

In Fig. 12 we show the MFs of the clusters which can be fitted by a single power-law. Because of the relatively large distance from the Sun (Table 1), the low-mass end of the observed MF of M 26 (panel a) occurs at $m \approx 0.98 M_{\odot}$, nearly the same value in which the MF break usually begins. Deeper observations are needed to access the MF break region in M 26. For M 93 (panel b) and NGC 3680 (panel d) the single powerlaw extends down to $\approx 0.7 M_{\odot}$ and $\approx 0.8 M_{\odot}$ respectively, with no evidence of a break. This is particularly true for the MF of NGC 5822 (panel c), which extends to $\approx 0.53 M_{\odot}$ fitted with a single power-law. The MF of NGC 188 is not shown in Fig. 12 because the distance from the Sun $\left(d_{\odot} \approx 1.7 \mathrm{kpc}\right)$ combined to the old age ( $\approx 7 \mathrm{Gyr}$ ) produce a MF restricted to the narrow mass range $0.9 \leq m\left(M_{\odot}\right) \leq 1.1$. The mass range is too narrow for any inferences on the presence of a MF break in this old open cluster.

Recall that the MFs of the present cluster sample have been built by taking into account the 2MASS $99.9 \%$ Completeness Limit (Sect. 3.1.3). Consequently, the low-mass end of each MF presented in this paper corresponds to the same apparent magnitudes, $J=15.8, H=15.1$ and $K_{\mathrm{S}}=14.3$. In this sense, if the MF break was artificially created by detection incompleteness in the faint magnitude limit, it should be present in the MFs of all clusters, irrespective of the corresponding low-mass limit. This is not the case of the MFs of M 26, M 93, NGC 5822 and NGC 3680 (Fig. 12), in which the low-mass limit varies from $\approx 0.5 M_{\odot}(\mathrm{NGC} 5822)$ to $\approx 1.0 M_{\odot}(\mathrm{M} 26)$.

The presence of the MF break is not associated to cluster age and mass or concentration parameter, at least in the studied clusters. However, the value of $m_{\text {break }}$ seems to decrease with cluster mass. We conclude that the MF break for $m \leq$ $1 M_{\odot}$ is definitively present in NGC 2516 , NGC $2287, \mathrm{M} 48$, NGC 2477, IC 4651 and M 67. On the other hand it is absent, at least for the mass range $m \geq 0.7 M_{\odot}$, in M93, NGC 5822 and NGC 3680. Further research accessing low-mass stars in a statistically significant sample of open clusters is necessary to settle the issue whether or not the MF break is present in all cases, and thus test the hypothesis of the universal character of Kroupa's IMF.

\subsection{Mass segregation and low-mass stars evaporation}

A comparison of the core and overall MF slopes in Table 3 may help us to evaluate the amount of mass segregation and low-mass stars evaporation which have already took place in the clusters. Except for M 26, the core MF slope in the remaining clusters is much flatter than the overall one. In the young and rather massive (Table 3) cluster M 26 both MF slopes correspond essentially to the standard Salpeter one, within uncertainties. This suggests that mass segregation and low-mass stars evaporation have not yet had time to produce observable changes neither in the internal structure of M26 nor in the MFs. This fact is consistent with the overall relaxation time $t_{\text {relax }} \sim 180 \mathrm{Myr}$, which is larger than the cluster age ( $\left.\sim 70 \mathrm{Myr}\right)$. On the other hand, the similarity between the core and overall MF slopes in M 26 occurs despite the much shorter core relaxation time of $t_{\text {relax }} \sim 3.2 \mathrm{Myr}$. This apparent contradiction reflects the complex way in which internal dynamical evolution relates to age and relaxation time, and in particular the different evolution time-scales associated to the core and overall regions.

In order to investigate the relationship between relaxation time and cluster age with dynamical evolution, and to estimate the corresponding effects on MFs we calculate for each cluster the evolutionary parameter $(\tau)$, which is defined as the ratio of the cluster age to the relaxation time, $\tau=$ age $/ t_{\text {relax }}$. We take advantage of the 2MASS spatial coverage to examine separately the evolution of the core and overall regions. In Table 5 we list $t_{\text {relax }}$ and $\tau$ for each cluster. 
Table 5. Relaxation time and evolutionary parameter.

\begin{tabular}{|c|c|c|c|c|}
\hline \multirow[b]{2}{*}{ Cluster } & \multicolumn{2}{|c|}{ Core } & \multicolumn{2}{|c|}{ Overall } \\
\hline & $\begin{array}{c}t_{\text {relax }} \\
(\mathrm{Myr})\end{array}$ & $\tau$ & \multicolumn{2}{|c|}{ (Myr) } \\
\hline (1) & (2) & (3) & (4) & (5) \\
\hline M26 & $3.2 \pm 2.0$ & $22 \pm 14$ & $178 \pm 116$ & $0.4 \pm 0.3$ \\
\hline NGC 2516 & $0.4 \pm 0.1$ & $365 \pm 74$ & $94 \pm 24$ & $1.7 \pm 0.4$ \\
\hline NGC 2287 & $0.8 \pm 0.2$ & $212 \pm 46$ & $40 \pm 10$ & $4.0 \pm 1.0$ \\
\hline M 48 & $0.7 \pm 0.2$ & $517 \pm 166$ & $48 \pm 28$ & $7.0 \pm 4.5$ \\
\hline M93 & $0.9 \pm 0.2$ & $457 \pm 112$ & $147 \pm 93$ & $2.7 \pm 1.7$ \\
\hline NGC 5822 & $2.4 \pm 1.3$ & $423 \pm 234$ & $262 \pm 170$ & $3.8 \pm 2.5$ \\
\hline NGC 2477 & $6.2 \pm 2.5$ & $181 \pm 75$ & $597 \pm 344$ & $1.9 \pm 1.0$ \\
\hline NGC 3680 & $0.2 \pm 0.1$ & $7100 \pm 2700$ & $29 \pm 17$ & $55 \pm 32$ \\
\hline IC 4651 & $0.6 \pm 0.1$ & $2854 \pm 455$ & $33 \pm 7$ & $54 \pm 13$ \\
\hline M 67 & $1.2 \pm 0.2$ & $2611 \pm 402$ & $80 \pm 15$ & $40 \pm 8$ \\
\hline NGC 188 & $14 \pm 9$ & $504 \pm 321$ & $631 \pm 400$ & $11 \pm 7$ \\
\hline
\end{tabular}

Table Notes. The relaxation time is calculated according to $t_{\text {relax }} \approx$ $0.12\left(\frac{N}{\ln N}\right)\left(\frac{R}{1 \mathrm{pc}}\right)\left(\frac{\sigma_{v}}{1 \mathrm{~km} \mathrm{~s}^{-1}}\right)^{-1}(\mathrm{Myr})$, where $N$ is the total number of stars, $R$ is the radius of the region considered and $\sigma_{v}$ is the velocity dispersion. We adopted $\sigma_{v} \approx 3 \mathrm{~km} \mathrm{~s}^{-1}$ (Binney \& Merrifield 1998). $\tau=$ age $/ t_{\text {relax }}$ is the evolutionary parameter.

Considering separately massive and less-massive clusters, we found that core and overall relaxation times are linearly related. For the massive clusters we derive the relation $t_{\text {relax }}($ overall $) \sim(89 \pm 15) \times t_{\text {relax }}($ core $)$ (correlation coefficient of $\mathrm{CC} \approx 0.94)$ and an average of $\sim 120$. For the less-massive ones $t_{\text {relax }}($ overall $) \sim(56 \pm 20) \times t_{\text {relax }}($ core $)(C C \approx 0.85)$ and an average of $\sim 80$.

We assume that flat overall MF slopes can be accounted for by low-mass stars evaporation resulting from large-scale mass segregation and external effects such as tidal stripping by the Galactic gravitational field. In this case, overall MF flattening is expected to be associated to $\tau$ (overall). In fact, in the clusters M 26, NGC 2516, NGC 2287, M 48, M 93, NGC 5822 and NGC 2477, which have overall MF slopes similar to that of Salpeter (thus, little low-mass stars evaporation), $\tau$ (overall) $\leq$ 7. Consequently, the age of these clusters results smaller than $\sim 7 \times t_{\text {relax }}$ (overall) which turns out to be too short a time for the evaporation to produce significant changes in the overall MFs. In this sense the flat core MFs of NGC 2516, NGC 2287, M 48, M 93, NGC 5822 and NGC 2477 can be accounted for by mass segregation, since for these clusters $\tau$ (core) $\geq 200$. The nearly Salpeter MF in the core of M 26 is probably due to the small $\tau($ core $) \sim 22$.

On the other hand, NGC 3680, IC 4651 and M 67, which have flat overall MF slopes, have $\tau$ (overall) $\geq 40$. The very flat core MFs in these clusters result from $\tau$ (core) $\geq 2600$. Thus, the core and overall evolutionary parameters are large enough for the large-scale mass segregation and low-mass stars evaporation to have produced significant MF flattening in these clusters.

Although with $\tau$ (overall) $\sim 11$ and $\tau$ (core) $\sim 504$, the oldest cluster in this work NGC 188 doesn't seem to fit in
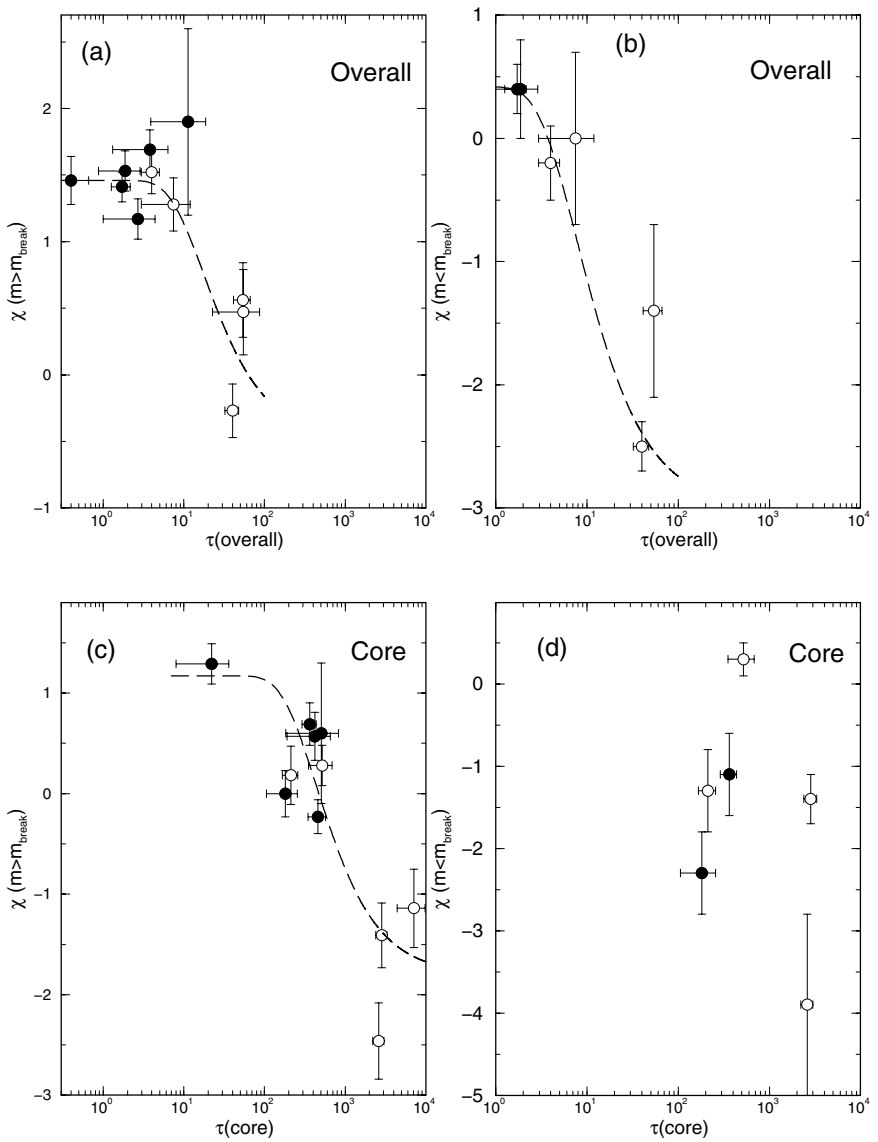

Fig. 13. MF slopes as a function of the evolutionary parameter $\tau$. Left panels: MFs in the range $m \geq m_{\text {break }}$; right panels: MFs in the range $m \leq m_{\text {break }}$. Top panels: the scale in the abscissa is based on the overall $\tau$; bottom panels: abscissa in terms of core $\tau$. Dashed lines in panels a)-c): tentative fit with $\chi(\tau)=\chi_{0}-\chi_{1} \exp \left(-\tau_{0} / \tau\right)$. Symbols as in Fig. 7.

the above scenario, because its overall MF slope is $\chi \sim 1.9$. However, because of the old age and large distance from the Sun we could access the MF only over the reduced range $0.9 M_{\odot}-1.0 M_{\odot}$, which produced large uncertainties in the MF slope.

We summarise the evolutionary scenario of core and overall MFs with respect to $\tau$ in Fig. 13. The overall MF slopes (for $m \geq m_{\text {break }}$ ) of the massive clusters (panel a) do not change significantly for $0.4 \leq \tau$ (overall) $\leq 11$. On the other hand, the overall MFs of the less-massive clusters begin to flatten for $\tau$ (overall) $>7$, which means that evaporation effects tend to become appreciable on the MFs when the age of the cluster becomes larger than $\sim 7 \times t_{\text {relax }}$ (overall). This occurs probably because of the looser nature of the less-massive clusters.

Because of high density and small dimensions (and thus small $\left.t_{\text {relax }}\right)$, core MFs tend to flatten because of mass segregation for $\tau$ (core) $>22$ (M 26) and $\tau$ (core) $\leq 150$, in massive and less-massive clusters alike (panel c). To first order we take $\tau($ core $)=100$ as representative of the value for which core MF flattening becomes significant.

Based on the discussion above we can express the time it takes for significant changes in the MFs (in the range $m \geq m_{\text {break }}$ ) to be produced as $\Delta t=\tau \times t_{\text {relax }}$. In the 
less-massive clusters the core and overall MFs begin to flatten at $\tau$ (core) $\sim 100$ and $\tau$ (overall) $\sim 7$, respectively. Taking into account the average ratio $t_{\text {relax }}($ overall $) / t_{\text {relax }}($ core $) \approx 80$, we find $\Delta t$ (overall) $\sim 6 \times \Delta t$ (core). We conclude that appreciable slope flattenings in the overall MFs of the less-massive clusters take on average $\sim 6$ times longer to occur than in the core MFs.

The value of $\tau$ (overall) in which the overall MFs of massive clusters begin to flatten is not clear in panel a of Fig. 13. Thus, assuming it as $\tau$ (overall) $\sim 11$ (NGC 188) for simplicity, and considering the average ratio $t_{\text {relax }}($ overall $) / t_{\text {relax }}($ core $) \approx 120$, we find that slope flattenings in the overall MFs of massive clusters take on average $\sim 13$ times longer to occur than in the core MFs.

In the mass range $m \leq m_{\text {break }}$ the overall MFs (panel b) flatten for $\tau$ (overall) $\geq 2$, which indicates that in this mass range dynamical evolution effects are effective in flattening the MFs quite rapidly. The scatter in panel d does not allow to draw conclusions on the behaviour of the core MFs.

The discussion above (and the apparent dependence of $\chi$ on $\tau$ in Fig. 13) suggests that the MF slopes undergo an exponential decay with $\tau$. Despite the reduced number of points we tentatively applied a fit with the empirical function $\chi(\tau)=$ $\chi_{0}-\chi_{1} \exp \left(-\tau_{0} / \tau\right)$ to the core and overall $\chi$ distributions (panels a-c in Fig. 13), combining massive and less-massive clusters. For $\chi$ (overall) we derived $\chi_{0}=1.46 \pm 0.10, \chi_{1}=$ $1.9 \pm 0.7$ and $\tau_{0}=18 \pm 14$, with a correlation coefficient $\mathrm{CC}=0.88$. For $\chi$ (core) we found $\chi_{0}=1.17 \pm 0.23, \chi_{1}=3.0 \pm$ 0.7 and $\tau_{0}=439 \pm 156$, with $\mathrm{CC}=0.88$. The resulting fits are shown in Fig. 13. For the overall $\chi$ in the range $m \leq m_{\text {break }}$ (panel $\mathrm{b}$ we derive $\chi_{0}=0.42 \pm 0.27, \chi_{1}=3.4 \pm 0.4$ and $\tau_{0}=8 \pm 4$, with $\mathrm{CC}=0.98$. These analytical relations can be used to compare core and overall MF flattening time scales. According to the above relations the overall MFs of the lessmassive clusters would flatten by $\Delta \chi=0.35$ in $\sim 4 \times$ the time it would take for the core MF to flatten by the same amount. In the massive clusters this ratio would be $\sim 6$. Both values basically agree with our previous estimates (see above).

In the same way, the overall MFs in the range $m \leq m_{\text {break }}$ flatten by $\Delta \chi=0.35$ in $\sim 1 / 3$ of the time the $m \geq m_{\text {break }}$ overall MFs flatten by the same amount. This is consistent with the fact that on the way towards energy equipartition, stars in the range $m \leq m_{\text {break }}$ should have, on average, a larger velocity dispersion $\left(\sigma_{\mathrm{v}} \sim 1 / \sqrt{m}\right)$ than those in the range $m \geq m_{\text {break }}$ and thus, they may leave out the cluster first.

A larger number of clusters is necessary to first check the existence of this analytical representation of $\chi(\tau)$ and then to derive more accurate values of $\chi_{0}, \chi_{1}$ and $\tau_{0}$, and finally, discuss further implications.

\section{Evidence of an open cluster fundamental plane}

In Sect. 4 we discussed relations involving pairs of intrinsic open cluster parameters. We found a few correlations among core and overall parameters, while in some plots massive and less-massive clusters are clearly segregated. This raises the question whether one of these pairs might be related to a third parameter, which could reflect the existence of a fundamental

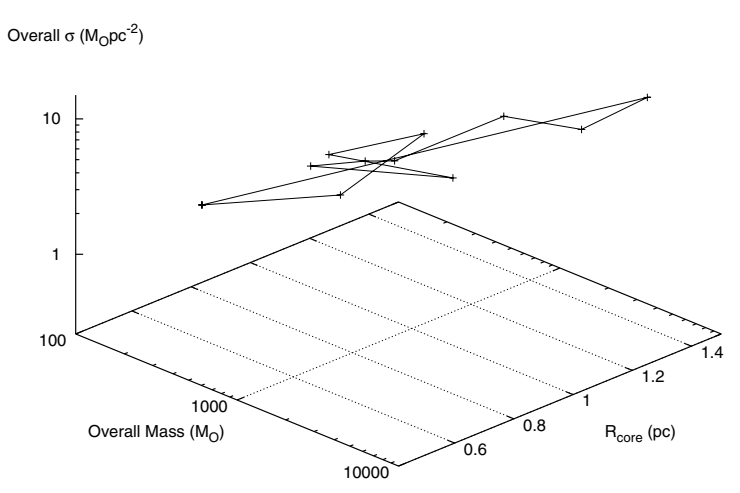

Fig. 14. Evidence of a fundamental plane of open cluster parameters. The distribution of overall mass ( $x$-axis), core radius ( $y$-axis) and projected overall mass density ( $z$-axis) essentially defines a plane in this $3 \mathrm{D}$ projection.

plane (FP) of open cluster parameters, similar to that of the elliptical galaxies (Dressler et al. 1987; Djorgovski \& Davis 1987). Elliptical galaxies populate a plane in the 3D-parameter space defined by the luminosity $(L)$, effective surface brightness $\left(\mu_{\mathrm{e}}\right)$ and velocity dispersion $\left(\sigma_{\mathrm{v}}\right)$. The plane comes about because as a consequence of the virial theorem a well-defined relationship between mass $(M), \mu_{\mathrm{e}}$ and $\sigma_{\mathrm{v}}$ is expected to occur in elliptical galaxies. Scatter in the plane depends essentially on the function relating the ratio $M / L$ with $L$ and $\mu_{\mathrm{e}}$ (Lucey et al. 1991). There is suggestive evidence that the planes of the ellipticals in the Coma cluster and that in the Virgo are not parallel (Lucey et al. 1991).

Although complete virialization in open clusters is an idealization, as clusters of different masses age they may reach advanced dynamical states. Indeed, kinetic theory and numerical simulations predict that $N$-body encounters in multi-mass models tend to produce energy equipartition within a timescale of the order of the relaxation time (de la Fuente Marcos \& de la Fuente Marcos 2002). Except in M 26, the overall relaxation times for the remaining clusters in the present work are significantly smaller than the cluster age. Core relaxation times are indeed much smaller than the ages (Table 5). In this sense, varying levels of relationship between open cluster parameters are expected to occur, particularly in the old clusters.

Open clusters do differ of elliptical galaxies particularly in terms of stellar population (absolute numbers and age), linear dimension and dynamical evolutionary state. Thus, the existence of an equivalent FP in open clusters raises some questions. (i) Is the open cluster FP related to dynamical evolution? (ii) Does the massive/less-massive cluster segregation (Sect. 4) still hold in the FP? (iii) Does Galactocentric distance affect the FP? A more comprehensive sample of open clusters is essential to answer these questions.

In the case of the present open clusters (Tables 2 and 3) we found that the overall mass and core radius are effectively related to the projected overall mass density. In Fig. 14 we show a 3D-projection of those three parameters, where one can see that they distribute nearly in a plane. A similar relation occurs for overall mass, core radius and projected core mass density. 
At the present stage the number of open cluster parameters is of the same order as the number of objects already analyzed by the techniques presented in the previous sections. We are using the same techniques to derive parameters of a larger number of clusters with significantly different properties, such as age, mass, core and limiting radii, densities, Galactocentric distance, etc. Our goal is to apply a principal component analysis on this comprehensive cluster sample not only to search for correlations among parameters, but to better explore the possibility of an open cluster FP as well.

\section{Concluding remarks}

We analysed 11 nearby open clusters with ages in the range 70 Myr to $7 \mathrm{Gyr}$ and masses in the range $\approx 400 M_{\odot}$ to $\approx 5300 M_{\odot}$ based on $J, H$ and $K_{\mathrm{S}}$ 2MASS photometry. The clusters are M26, NGC 2516, NGC 2287, M 48, M 93, NGC 5822, NGC 2477, NGC 3680, IC 4651, M 67 and NGC 188. Radial density profiles and mass functions were built after taking into account the field contamination. As a consequence we derived a homogeneous set of parameters associated to the structure and stellar and dynamical evolution of the clusters.

The method is illustrated in detail by analysing the populous open clusters NGC 2477 and NGC 2516 for the first time in the near-infrared. For NGC 2477 we derive an age of $1.1 \pm$ $0.1 \mathrm{Gyr}$, distance from the Sun $d_{\odot}=1.2 \pm 0.1 \mathrm{kpc}$, core radius $R_{\text {core }}=1.4 \pm 0.1 \mathrm{pc}$, limiting radius $R_{\lim }=11.6 \pm 0.7 \mathrm{pc}$ and total mass (extrapolating the MF down to the H-burning mass limit, $\left.0.08 M_{\odot}\right) m_{\text {tot }} \approx(5.3 \pm 1.6) \times 10^{3} M_{\odot}$. The MF slope varies significantly in this cluster, being flat $(\chi \approx 0.0)$ in the core $(0.0 \leq r(\mathrm{pc}) \leq 1.4)$ and steep $(\chi \approx 3.3)$ in the outskirts $(5.2 \leq r(\mathrm{pc}) \leq 11.7)$. The overall MF has a slope $\chi \approx 1.5$. The number-density of evolved stars (with respect to MS stars with mass down to $0.08 M_{\odot}$ ) in the core is about 4 times as large as in the cluster as a whole. In the halo the numberdensity of low-mass stars is $\sim 4$ times as large as that of the evolved stars. These facts reflect the effects of large-scale mass segregation in NGC 2477. For NGC 2516 we derive an age of $160 \pm 10 \mathrm{Myr}, d_{\odot}=0.44 \pm 0.02 \mathrm{kpc}, R_{\text {core }}=0.6 \pm 0.1 \mathrm{pc}$, $R_{\mathrm{lim}}=6.2 \pm 0.2 \mathrm{pc}$ and $m_{\mathrm{tot}} \approx(1.3 \pm 0.2) \times 10^{3} M_{\odot}$. Similarly to NGC 2477, mass-segregation effects in NGC 2516 are reflected in the spatial variation of the MF slopes, $\chi \approx 0.7$ in the core $(0.0 \leq r(\mathrm{pc}) \leq 0.6)$ and $\chi \approx 1.8$ in the outskirts $(1.6 \leq r(\mathrm{pc}) \leq 6.2)$. The overall MF has a slope $\chi \approx 1.4$. The overall MFs of NGC 2477 and NGC 2516 have slopes similar to a standard Salpeter IMF $(\chi \approx 1.35)$.

Six of the 11 clusters present a break around $1 M_{\odot}$ followed by a sharp flattening in the MF in the mass range $\approx 0.5 M_{\odot}$ to $\approx 1 M_{\odot}$. The mass range where we detected the MF break in open clusters basically coincides with previous results based on star counts in the solar neighbourhood (Kroupa 2001, 2002). In the clusters analysed here the MF break is not associated to mass or concentration parameter. The presence of the MF break in all clusters would be consistent with the universal character of the IMF (Kroupa 2002). However, 3 clusters in our sample do not present the MF break, at least for the mass range $m \geq 0.7 M_{\odot}$. This does not exclude the possibility that in some clusters the MF break occurs at lower masses or that dynamical effects may somehow damp out features in the MFs. To settle these issues we are analyzing a more statistically significant sample of open clusters.

We investigated the dependence of MF slopes on the evolutionary parameter $\tau=$ age $/ t_{\text {relax }}$. We found that dynamical effects begin to produce substantial flattening in the core MFs of massive and less-massive clusters when the cluster age is $\sim 100 \times t_{\text {relax }}$ (core). In the overall MFs the changes become noticeable when $\sim 7 \times t_{\text {relax }}$ (overall) and $\sim 11 \times t_{\text {relax }}$ (overall), respectively for the less-massive and massive clusters. Considering the linear relationship between core and overall relaxation times, we conclude that appreciable slope flattening in the overall MFs of the less-massive clusters take $\sim 6$ times longer to occur than in the core MFs. In the massive clusters they take a time $\sim 13$ times longer. We found that MF slopes vary with $\tau$ according to the empirical relation $\chi(\tau)=\chi_{0}-\chi_{1} \exp \left(-\tau_{0} / \tau\right)$

We also searched for relations of cluster parameters with cluster age and Galactocentric distance. The main results are: (i) cluster size correlates both with cluster age and Galactocentric distance; (ii) because of size and mass scaling, core and limiting radii, and core and overall mass correlate as well; (iii) core radius and overall mass are correlated, but massive $\left(m \geq 1000 M_{\odot}\right)$ and less-massive clusters follow separate, nearly parallel paths on the plane; (iv) the MF slopes of massive clusters are restricted to a narrow range, while those of the less-massive clusters distribute over a wider range; $(v)$ core and overall MF slopes are correlated.

We found relations involving three parameters simultaneously which may suggest a fundamental plane of open cluster parameters. The relations involve overall mass, core radius, and projected overall mass density or projected core mass density, which are those more closely related to the parameters involved in the Fundamental Plane of ellipticals.

As prospective work we are carrying on the present analysis for a larger sample in order to draw more quantitative conclusions on the fraction of open clusters which present a break in the MF, the reason why some do not have the break, and on correlations of $m_{\text {break }}$ with other cluster parameters, further exploring as well the possibility of a fundamental plane.

Acknowledgements. We thank the anonymous referee for helpful suggestions. This publication makes use of data products from the Two Micron All Sky Survey, which is a joint project of the University of Massachusetts and the Infrared Processing and Analysis Center/California Institute of Technology, funded by the National Aeronautics and Space Administration and the National Science Foundation. We also made use of the WEBDA open cluster database. We acknowledge support from the Brazilian Institution CNPq.

\section{References}

Battinelli, P., Brandimarti, A., \& Capuzzo-Dolcetta, R. 1994, A\&AS, 104,379

Bergond, G., Leon, S., \& Guibert, J. 2001, A\&A, 377, 462

Bica, E., \& Bonatto, C. J. 2005, A\&A, 431, 943

Bica, E., Bonatto, C. J., \& Dutra, C. M. 2004a, A\&A, 405, 991

Bica, E., Bonatto, C. J., \& Dutra, C. M. 2004b, A\&A, 422, 555

Binney, J., \& Tremaine, S. 1987, in Galactic Dynamics (Princeton, NJ: Princeton University Press, Princeton series astrophys.) 
Binney, J., \& Merrifield, M. 1998, in Galactic Astronomy (Princeton, NJ: Princeton University Press, Princeton series astrophys.)

Bonatto, C., \& Bica, E. 2003, A\&A, 405, 525

Bonatto, C. J., Bica, E., \& Pavani, D. B. 2004a, A\&A, 427, 485

Bonatto, C., Bica, E., \& Girardi, L. 2004b, A\&A, 415, 571

Bonatto, C. J., Bica, E., \& Santos, Jr., J. F. C. 2005, A\&A, in press

Djorgovski, S., \& Davis, M. 1987, ApJ, 313, 59

Dressler, A., Lynden-Bell, D., Burstein, D., et al. 1987, ApJ, 313, 42

Dutra, C. M., Santiago, B. X., \& Bica, E. 2002, A\&A, 381, 219

Eigenbrod, A., Mermilliod, J.-C., Clariá, J. J., Andersen, J., \& Mayor, M. 2004, A\&A, 423, 189

Friel, E. D. 1995, ARA\&A, 33, 381

de la Fuente Marcos, R. 1998, A\&A, 333, L27

de la Fuente Marcos, R., \& de la Fuente Marcos, C. 2002, Ap\&SS, 280, 381

Girardi, L., Bertelli, G., Bressan, A., et al. 2002, A\&A, 391, 195

von Hippel, T., Gilmore, G., Tanvir, N., Robinson, D., \& Jones, D. H. P. 1996, AJ, 112, 192

Hurley, J. R., Tout, C. A., Aarseth, S. J., \& Pols, O. R. 2001, MNRAS, 323,630

Hut, P. 1996, in The Origins Evolutions, and Destinies of Binary Stars in Clusters, ed. E. F. Milone, \& J.-C. Mermilliod, ASP Conf. Ser., 90,391

Janes, K., Tilley, C., \& Lyngå, G. 1988, AJ, 95, 771

Jeffries, R. D., Thurston, M. R., \& Hambly, N. C. 2001, A\&A, 375, 863

King, I. 1962, AJ, 67, 471

King, I. 1966a, AJ, 71, 64
King, I. 1966b, AJ, 71, 276

Kroupa, P. 2001, MNRAS, 322, 231

Kroupa, P. 2002, Science, 295, 82

Lucey, J. R., Bower, R. G., \& Ellis, R. S. 1991, MNRAS, 249, 755

Lyngå, G. 1982, A\&A, 109, 213

Massey, P. 1998, in The Stellar Initial Mass Function, ed. G. Gilmore, D. Howell (San Francisco: Astronomical Society of the Pacific), ASP Conf. Ser., 142, 17

Meibom, S., Andersen, J., \& Nordström, B. 2002, A\&A, 386, 187

Mermilliod, J. C. 1996, in The Origins, Evolution, and Destinies of Binary Stars in Clusters, ed. E. F. Milone, \& J.-C. Mermilliod, ASP Conf. Ser., 90, 475

Montgomery, K. A., Marschall, L. A., \& Janes, K. A. 1993, AJ, 106, 181

Nilakshi, S. R., Pandey, A. K., \& Mohan, V. 2002, A\&A, 383, 153

Reid, M. J. 1993, ARA\&A, 31, 345

Salpeter, E. 1955, ApJ, 121, 161

Sarajedini, A., von Hippel, T., Kozhurina-Platais, V., \& Demarque, P. 1999, AJ, 118, 2894

Scalo, J. M. 1986, Fundam. Cosmic. Phys., 11, 1

Skrutskie, M., Schneider, S. E., Stiening, R., et al. 1997, in The Impact of Large Scale Near-IR Sky Surveys, ed. Garzon et al. (Netherlands: Kluwer), 210, 187

Soares, J. B., \& Bica, E. 2002, A\&A, 388, 172

Tadross, A. L., Werner, P., Osman, A., \& Marie, M. 2002, NewAst, 7, 553

Trager, S. C., King, I. R., \& Djorgovski, S. 1995, AJ, 109, 218 